\title{
Origin of the Reissner-Nordström-de Sitter instability
}

\author{
Oscar J.C. Dias $\circledast^{1, *}$ and Jorge E. Santos ${ }^{2,3, \dagger}$ \\ ${ }^{1}$ STAG Research Centre and Mathematical Sciences, Highfield Campus, \\ University of Southampton, Southampton SO17 1BJ, United Kingdom \\ ${ }^{2}$ Department of Applied Mathematics and Theoretical Physics, University of Cambridge, \\ Wilberforce Road, Cambridge CB3 OWA, United Kingdom \\ ${ }^{3}$ Institute for Advanced Study, Princeton, New Jersey 08540, USA
}

(Received 19 August 2020; accepted 24 November 2020; published 17 December 2020)

\begin{abstract}
We give strong numerical evidence for the existence of an instability afflicting six-dimensional ReissnerNordström de Sitter (RNdS) black holes. This instability is akin of the Konoplya-Zhidenko instability present in RNdS black holes in seven spacetime dimensions and above. Moreover, we perform a detailed analysis of the near-horizon limit of extremal RNdS black holes, and find that, typically, unstable gravitational modes effectively behave as a massive scalar field whose mass violates the $\mathrm{AdS}_{2}$ Breitenlöhner-Freedman bound (if and only if $d \geq 6$ ), thus providing a physical argument for the existence of the instability. Finally, we show that the frequency spectrum of perturbations of RNdS has a remarkable intricate structure with several bifurcations/mergers that appears unique to RNdS black holes.
\end{abstract}

DOI: 10.1103/PhysRevD.102.124039

\section{INTRODUCTION}

Kodama and Ishibashi proved that Reissner-Nordström de Sitter (RNdS) black holes in $d=4$ and $d=5$ spacetime dimensions are linearly mode stable [1]. Therefore, the finding by Konoplya and Zhidenko [2,3], that RNdS black holes can be unstable to gravitational perturbations if they live in $d \geq 7$ spacetime dimensions $(n=d-2 \geq 5)$, came with some surprise. The existence of this instability was further confirmed in [4] where it was also noted that there is a simple (necessary but not sufficient) criterion-originally due to [5] - that predicts the existence of the instability. Essentially, [5] proposes that a system should be unstable whenever an integral (between the event and cosmological horizons) of the Schrödinger potential of the perturbation is negative. The instability of [2] is in these conditions [4]. More recently, this RNdS instability was also studied in the framework of the large $d$ limit of general relativity [6].

There are however some fundamental questions that are left unanswered by $[2-4,6]$ and that we address in this manuscript. First, we would like to understand the physical origin of this instability, and, in particular, why it only appears in higher dimensions. This is particularly

\footnotetext{
ojcd1r13@soton.ac.uk

jss55@cam.ac.uk
}

Published by the American Physical Society under the terms of the Creative Commons Attribution 4.0 International license. Further distribution of this work must maintain attribution to the author(s) and the published article's title, journal citation, and DOI. Funded by SCOAP. important, since for $d=4$ and $d=5$ Kodama and Ishibashi proved linear-mode stability [1]. It is also important in the context of recent studies on strong cosmic censorship violation in RNdS black holes [7-18]. Second, $[2,4]$ find that the system is unstable for $d \geq 7$ but their analysis leaves the stability properties of the $d=6$ case undetermined. Again, stability is proven only for $d=4,5$ so could it be that there is an instability also for $d=6$ ?

In this paper we address these two questions. In Sec. II we start by reviewing the RNdS black holes and the relevant sector of perturbations that can be unstable. Then, in Sec. III we point out that there is a criterion for instability that predicts and, at the same time, justifies an instability in RNdS black holes. This instability criterion was conjectured by Durkee and Reall [19] and later proved by Hollands and Wald [20]. Strictly speaking it was proven only for nonpositive cosmological constant backgrounds, $\Lambda \leq 0$, but, as argued in [20], it should also hold for de Sitter backgrounds $(\Lambda>0)$. Applied to our system, this Durkee-Reall instability criterion essentially states that one should take the near-horizon limit of the gravitational perturbation master equation about the extremal RNdS black hole. After the limit is taken, the gravitational master equation effectively reduces to a Klein-Gordon equation for a massive scalar field in an $\mathrm{AdS}_{2}$ background. If this near-horizon effective mass is smaller than the $\mathrm{AdS}_{2}$ Breitenlöhner-Freedman (BF) mass bound then the full RNdS extremal geometry should be unstable. By continuity, this instability should extend away from extremality. As shown in Sec. III, this criterion predicts the existence of an instability for RNdS black holes in $d \geq 6$. In Sec. IV, we 
numerically solve the perturbation master equation and we confirm that the instability is indeed present for $\mathrm{RNdS}$ black holes in $d \geq 6$ dimensions. Therefore, the two main outcomes of our study are the following: (1) we provide a physical origin of the Konoplya-Zhidenko instability (violation of the $\mathrm{AdS}_{2} \mathrm{BF}$ bound) and (2) we establish the presence of the instability in $d=6$.

Adding to these two main results, we will also take the opportunity to explore in detail the properties of the instability. For example, we will establish that the instability is present in a broader region of the parameter space than originally reported in [2-4]. Indeed, in one of our studies we will look for the instability directly in the extremal configuration and will find that all extremal RNdS black holes are unstable for $d \geq 6 .{ }^{1}$ That is, [2-4] identified the presence of the instability only for large values of $r_{+} / r_{c}$ (where $r_{+}$and $r_{c}$ are the event and cosmological horizons of RNdS) but we find that the instability is actually present in the whole range $\left.r_{+} / r_{c} \in\right] 0,1[$ for extremal RNdS. Our findings are not in conflict with the Durkee-Reall instability criterion, since the latter is known to be a sufficient, but not necessary, condition for the existence of an instability. Additionally, we will also analyze the properties of the instability away from extremality and we will also look directly for the onset of the instability. Finally, we will also study in some detail the quasinormal mode structure of the perturbations as we span the two-dimensional parameter space of RNdS black holes. We will find an intricate network of quasinormal mode branches with interesting bifurcations/mergers that, to the best of our knowledge, seem to be unusual in black hole perturbations (at least in $\Lambda \leq 0$ backgrounds).

\section{REISSNER-NORDSTRÖM DE SITTER BLACK HOLE AND ITS PERTURBATIONS}

We work with the Einstein-Maxwell theory, in $d=n+2$ spacetime dimensions $(n \geq 2)$, with a positive cosmological constant $\Lambda$ described by the action

$$
\begin{aligned}
S & =\frac{1}{16 \pi G} \int \mathrm{d}^{n+2} x \sqrt{-g}\left(R-2 \Lambda-F^{2}\right), \quad \text { with } \\
\Lambda & \equiv \frac{n(n+1)}{2 L^{2}}
\end{aligned}
$$

and where $R$ is the Ricci scalar of the metric $g, L$ is the de Sitter length scale, and $F=\mathrm{d} A$ is the Maxwell field strength associated with the Maxwell potential $A$.

A known solution of this theory is the RNdS black hole. In static coordinates, the gravitational and electric fields of this solution with mass $M$ and charge $q$ parameters are

\footnotetext{
${ }^{1}$ For $d=6$, the evidence is substantially weaker, as the instability growth rates are very small.
}

$$
\mathrm{d} s^{2}=-f \mathrm{~d} t^{2}+\frac{\mathrm{d} r^{2}}{f}+r^{2} \mathrm{~d} \Omega_{n}^{2}, \quad A=-\frac{q}{r} \mathrm{~d} t,
$$

with $\mathrm{d} \Omega_{n}^{2}$ being the line element of a unit radius $S^{n}$ and

$f(r)=1-\frac{r^{2}}{L^{2}}-\frac{2 M}{r^{n-1}}+\frac{Q^{2}}{r^{2(n-1)}}, \quad Q=\frac{\sqrt{2} q}{\sqrt{n(n-1)}}$.

For an appropriate range of parameters, specified below, $f$ has three real positive roots $r_{-} \leq r_{+} \leq r_{c}$ corresponding to the Cauchy horizon $\mathcal{C H}$, event horizon $\mathcal{H}^{+}$, and cosmological horizon $\mathcal{H}_{C}$, respectively. We can express $M$ and $L$ in terms of $r_{+}, r_{c}$ and $q$. The temperature of the event and cosmological horizons are, respectively, given by $T_{+}=\frac{f^{\prime}\left(r_{+}\right)}{4 \pi}$ and $T_{c}=-\frac{f^{\prime}\left(r_{c}\right)}{4 \pi}$.

When $T_{+}=0$ we have an extremal RNdS black hole. This happens for $q=q_{\mathrm{ext}}$ with

$$
\begin{gathered}
\frac{q_{\mathrm{ext}}}{r_{c}^{n-1}}=y_{+}^{n-1} \sqrt{\frac{n(n-1)}{2}} \sqrt{\frac{2 y_{+}^{n+1}-(n+1) y_{+}^{2}+n-1}{(n+1) y_{+}^{2 n}-2 n y_{+}^{n+1}+n-1}}, \\
\text { and } y_{+} \equiv \frac{r_{+}}{r_{c}} .
\end{gathered}
$$

The Einstein-Maxwell equations of motion are invariant under the scaling $g \rightarrow \lambda^{2} g, A \rightarrow \lambda A$, and $L \rightarrow \lambda L$, with $\lambda \in \mathbb{R}$, which we can use to construct dimensionless quantities in units of $r_{c}$. Therefore, we choose to parametrize the RNdS solution using the dimensionless parameters $q / q_{\text {ext }}$ and $y_{+}$.

We are interested on gravitoelectromagnetic perturbations of RNdS. These were studied in detail by KodamaIshibashi in [1]. Perturbations of (2.2) can be analyzed according to how they transform under diffeomorphisms of the $S^{n}$ sphere. There are a total of three families of perturbations that decouple from each other, namely, the scalar, vector, and tensor perturbations. These perturbations are built from scalar, vector, and tensor harmonics on $S^{n}$, respectively. We are primarily interested in scalar perturbations, which are built from spherical harmonics $S_{\ell}(\vec{x})$, where $\vec{x}$ collectively parametrize coordinates on the $n$ sphere. These harmonics are such that

$$
\square_{S^{n}} S_{\ell}(\vec{x})=\lambda_{S} S_{\ell}(\vec{x})
$$

with $\lambda_{S}=\ell(\ell+n-1)$ and $\ell \geq 0$ being an integer. Modes with $\ell=0,1$ were shown to be pure gauge in [1]. In particular, modes with $\ell=0$ describe changes in the mass of the background $\mathrm{RNdS}$ black hole while $\ell=1$ modes represent translations. Onwards, we shall take $\ell \geq 2$.

In the Schwarzschild limit, $q=0, \Phi_{\ell}^{\mathrm{S}-}(t, r)$ and $\Phi_{\ell}^{\mathrm{S}+}(t, r)$ describe, respectively, purely gravitational and purely electromagnetic perturbations [1]. However, when 
$q \neq 0$ the gravito-electromagnetic perturbations are coupled. As described in [1], one can introduce a separation anstaz of the form

$$
\Phi_{\ell}^{\mathrm{S}-}(t, r)=e^{-i \omega t} S_{\ell}(\vec{x}) \Phi_{\omega \ell}^{-}(r)
$$

which introduces the frequency $\omega$. One can then manipulate the Einstein and Maxwell equation to find a decoupled ordinary differential equation for the radial master field $\Phi_{\omega \ell}^{-}(r)$ [see Eq. (5.59) of [1] ], namely,

$$
f\left(f \Phi_{\omega \ell}^{-}(r)^{\prime}\right)^{\prime}+\left(\omega^{2}-V_{-}\right) \Phi_{\omega \ell}^{-}(r)=0
$$

where the potential $V_{-}\left(r ; n, r_{+}, r_{c}, q, \ell\right)$ can be found in Eqs. (5.61)-(5.63) of [1].

\section{NEAR-HORIZON CRITERION FOR INSTABILITY}

As reviewed below, the near-horizon limit of an extremal $\mathrm{RNdS}$ black hole is described by the direct product spacetime $\mathrm{AdS}_{2} \times S^{n}$. One of the main observations of the current manuscript is that the existence of some instabilities of the full RNdS black hole can be inferred from studying the behavior of the perturbation equation in the near horizon limit. More concretely, in the near-horizon limit the Kodama-Ishibashi master equation (2.7) reduces to an equation that has the form of a Klein-Gordon equation for a massive scalar field in $\mathrm{AdS}_{2}$. We will confirm that, according to the Durkee-Reall criterion [19,20], when this near-horizon effective mass violates the $\mathrm{AdS}_{2}$ Breitenlöhner-Freedman mass bound then the full RNdS geometry is unstable. ${ }^{2}$ This happens for $d \geq 6(n \geq 4)$. We will find that the $\mathrm{AdS}_{2} \mathrm{BF}$ bound violation gives a sufficient (but not necessary) criterion for the presence of an instability and also justifies its origin.

To get the near-horizon geometry of the extremal RNdS black hole, one first takes (2.2) with $q=q_{\mathrm{ext}}$ and zooms in around the event horizon region by making the coordinate transformations:

$$
\begin{aligned}
r & =r_{+}+\varepsilon \rho, \quad t=L_{2}^{2} \frac{\tau}{\varepsilon} ; \quad \text { with } \\
L_{2}^{2} & =\frac{r_{+}^{2}\left[2 n y_{+}^{n+1}-(n+1) y_{+}^{2 n}-n+1\right]}{(n-1)\left[-4 n y_{+}^{n+1}+(n+1)\left(y_{+}^{2 n}+n y_{+}^{2}\right)-(n-1)^{2}\right]} .
\end{aligned}
$$

The near-horizon solution is then obtained by taking $\varepsilon \rightarrow 0$ yielding [after a $U(1)$ gauge transformation]

\footnotetext{
${ }^{2}$ Strictly speaking the Durkee-Reall conjecture was originally formulated and proved only for $\Lambda \leq 0$ backgrounds [19,20] but, as argued in [20], it should also hold for $\Lambda>0$.
}

$$
\begin{aligned}
d s_{N H}^{2} & =L_{2}^{2}\left(-\rho^{2} d \tau^{2}+\frac{d \rho^{2}}{\rho^{2}}\right)+r_{+}^{2} d \Omega_{n}^{2}, \\
A_{\mu}^{N H} d x^{\mu} & =\alpha \rho d \tau, \quad \alpha=\frac{n-1}{\sqrt{2}} L_{2} \sqrt{1+(n-1) \frac{L_{2}^{2}}{r_{+}^{2}}} .
\end{aligned}
$$

This geometry is the direct product of $\mathrm{AdS}_{2} \times \mathrm{S}^{n}$ and has a Maxwell potential that is linear in the radial direction. This limiting solution is still a solution of the $(n+2)$-dimensional Einstein-Maxwell-dS theory. On the other hand, the $\mathrm{AdS}_{2}$ metric solves the two-dimensional Einstein-anti-de Sitter equations with $\mathrm{AdS}_{2}$ radius $L_{2}$, $R_{\mu \nu}=-L_{2}^{-2} g_{\mu \nu}$.

One can now take the near-horizon limit (3.1), together with $\omega=\varepsilon L_{2}^{-2} \tilde{\omega}$ directly on the gravitational master equation (2.7). After taking $\varepsilon \rightarrow 0$ this yields

$$
\left(\square_{2}-\mu^{2} L_{2}^{2}\right) \Phi_{\omega \ell}^{-}(\rho)=0,
$$

where $\square_{2}$ is the d'Alembertian in $\operatorname{AdS}_{2}$ and $\mu^{2}=$ $\mu^{2}\left(n, y_{+}, \ell\right)$ is the near-horizon effective mass of the system. Its particular expression is long and not enlightening so we do not reproduce it here. It depends on the harmonic number $\ell$, on the dimension $d=n+2$ and on $y_{+}$. The key point is that this near-horizon analysis is expected to provide a criterion for instability $[19,20]$ : whenever this mass is smaller than the $\mathrm{AdS}_{2} \mathrm{BF}$, $\mu_{\mathrm{BF}}^{2} L_{2}^{2}=-1 / 4$, one should have an instability of the full $\mathrm{RNdS}$ black hole. We further expect this instability to be the one found in $[2,4,6]$ (for $d \geq 7$ ). If so, the violation of the $\mathrm{AdS}_{2} \mathrm{BF}$ bound effectively explains the origin of the instability found in $[2,4,6]$.

In Fig. 1 we set $\ell=2$ and plot $\left(\mu^{2}-\mu_{\mathrm{BF}}^{2}\right) L_{2}^{2}$ as a function of $y_{+}=r_{+} / r_{c}$ for $n=4,5,6,7,8,9$ (see plot legends). We conclude that for $n \geq 4$ there are always values of $y_{+}$where $\left(\mu^{2}-\mu_{\mathrm{BF}}^{2}\right) L_{2}^{2}<0$ and thus for which the $\mathrm{AdS}_{2} \mathrm{BF}$ bound is violated and an instability should be present. Note that this includes the $d=6(n=4)$ case, which was also analyzed in $[2,4]$, but for which no instability was found. For $n=2,3$, i.e., $d=4,5$, one always has $\mu^{2}>\mu_{\mathrm{BF}}^{2}$ and we do not display these curves in Fig. 1. This result is consistent with the fact that $d=4,5 \mathrm{RNdS}$ are stable to all linear-mode gravitational perturbations [1].

In the next section we will confirm that the $\mathrm{AdS}_{2} \mathrm{BF}$ bound violation observed in Fig. 1 (for $n \geq 4$ ) indeed gives a sufficient criterion for the presence of an instability and also justifies its origin. But we will also show that this condition is not a necessary condition.

So far we discussed only harmonics with $\ell=2$. But we can also ask what happens if we consider modes with $\ell \geq 3$. Typically, we find that as the integer $\ell$ increases it becomes harder to get negative $\left(\mu^{2}-\mu_{\mathrm{BF}}^{2}\right) L_{2}^{2}$. For example, 


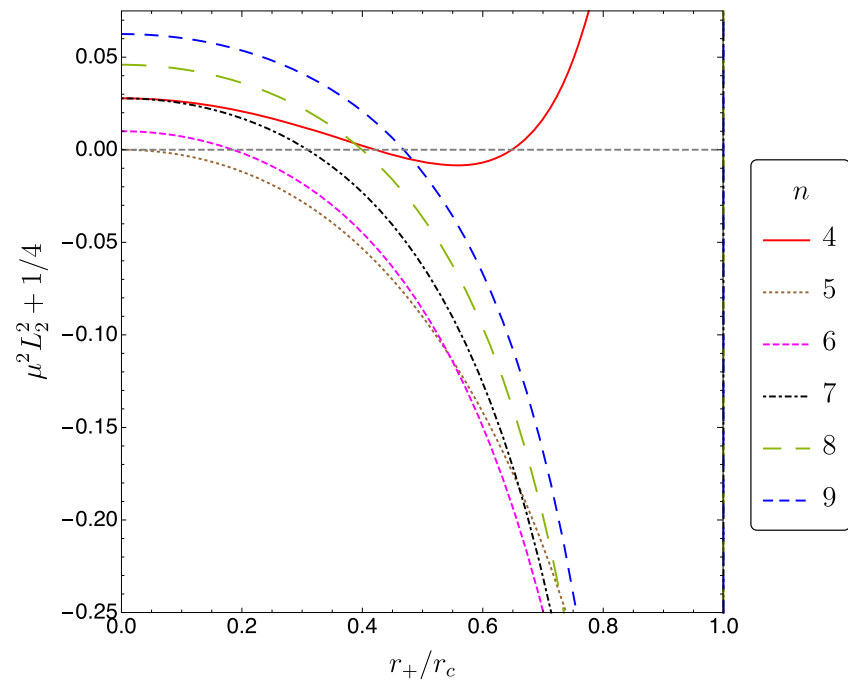

FIG. 1. Difference between the effective near-horizon $\mathrm{AdS}_{2}$ mass $\mu^{2} L_{2}^{2}$ and the $\mathrm{AdS}_{2} \mathrm{BF}$ bound $\mu_{B F}^{2} L_{\mathrm{AdS}}^{2}=-1 / 4$ as a function of $r_{+} / r_{c}$. When this quantity is negative one should expect an instability $[19,20]$.

in $n=4$ or $n=5$ dimensions, for $\ell \geq 3$ the $\mathrm{AdS}_{2} \mathrm{BF}$ bound is no longer violated for any $y_{+}$. As another example, for $n=6,7$ the harmonics $\ell=2,3$ generate a violation of the $\mathrm{AdS}_{2} \mathrm{BF}$ bound but this is no longer the case for $\ell \geq 4$. As a final example, for $n=8,9$ the harmonics $\ell=2,3,4$ generate a violation of the $\mathrm{AdS}_{2} \mathrm{BF}$ bound but this is no longer the case for $\ell \geq 5$.

\section{NUMERICAL RESULTS FOR THE INSTABILITY}

\section{A. Setup of the problem}

Our aim is to solve the Kodama-Ishibashi linear ordinary differential equation (ODE) (2.7), subject to relevant boundary conditions, to find the properties of linear mode perturbations in RNdS. More concretely, we want to fix the spacetime dimension $n$ and the RNdS black holedescribed by the dimensionless quantities $\left\{y_{+}, q / q_{\text {ext }}\right\}-$ as well as the harmonic quantum number $\ell$ and look for modes that are unstable.

To achieve our aims we have followed a strategy that is anchored on three main studies:

(i) Generically, we have a nonextremal RNdS black hole with $q / q_{\text {ext }}<1$. We can solve (2.7) as a quadratic eigenvalue problem for $\omega$ to find the eigenvalue/eigenfunction pair $\left(\omega r_{c}, \Phi_{\omega \ell}^{-}\right)$for a given set of $\left\{n, y_{+}, q / q_{\text {ext }}, \ell\right\}$. For a fixed $\{n, \ell\}$ we can then repeat the analysis and scan the full twodimensional parameter space of RNdS black holes.

(ii) As argued in Sec. III, the instability has a nearhorizon/extremal origin. So, when present, it should make its appearance at extremality and then extend away from extremality until it eventually shuts down. Therefore, we first consider the RNdS with $q=q_{\text {ext }}$ and solve (2.7) as a quadratic eigenvalue problem for the frequency to find the eigenpair $\left(\omega r_{c}, \Phi_{\omega \ell}^{-}\right)$, for a given $\left\{n, y_{+}, \ell\right\}$ directly at extremality.

(iii) Alternatively, instead of looking for the instability timescale, we can search directly for the onset of the instability whereby the frequency vanishes, $\omega=0$. To find this instability threshold, we solve (2.7) as a nonlinear eigenvalue problem for the black hole charge to find the onset charge $q=q^{\text {onset }}$ above which RNdS is unstable.

The above three strategies are complementary. In particular, the two-dimensional surface in the plot $\left\{y_{+}, q / q_{\mathrm{ext}}, \operatorname{Im}\left(\omega r_{c}\right)\right\}$ generated using the first study must (i) have the extremal curve with $q / q_{\text {ext }}=1$ obtained in the second study as a boundary line and (ii) intersect the onset curve $\omega=0$ of the third study when $\operatorname{Im}\left(\omega r_{c}\right)=0$. So the three complementary studies also provide nontrivial independent checks of the numerical results we obtain.

To proceed, one needs to use a numerical scheme to solve our boundary value problems. For that it is good to first introduce a new compact radial coordinate, namely,

$$
y=\sqrt{1-\sqrt{\frac{r_{c}-r}{r_{c}-r_{+}}}},
$$

such that $y=0$ describes the black hole horizon, $r=r_{+}$, and $y=1$ marks the location of the cosmological horizon, $r=r_{c}$.

Next, one needs to discuss the issue of the boundary conditions. These boundary conditions are different for the three eigenvalue problems I-III listed above and we discuss them separately.

Consider first the eigenvalue problem I. We have a nonextremal black hole and deformations propagate between the event and cosmological horizons where we impose as boundary conditions that the perturbations are regular in Eddington-Finkelstein coordinates. In particular, we take the modes to be ingoing at the event horizon and outgoing at the cosmological horizon. To find these boundary conditions we first do a Frobenius analysis about $y=0$ to find that

$$
\Phi_{\omega \ell}^{-}(y) \simeq y^{ \pm \frac{i \tilde{\omega}}{2 \pi \tilde{T}_{+}}}[1+\mathcal{O}(y)]
$$

where $T_{+}$is the event horizon temperature (its surface gravity divided by $2 \pi$ ) as defined below (2.3) and the tilde (here and in other expressions) is used to state that the quantities are measured in units of $r_{c}$, e.g.,

$$
\tilde{\omega} \equiv \omega r_{c}, \quad \tilde{T}_{+} \equiv T_{+} r_{c}, \quad \tilde{T}_{c} \equiv T_{c} r_{c} .
$$

Ingoing boundary conditions at the black hole horizon requires choosing the lower sign in (4.2). A similar analysis around the cosmological horizon, $y=1$, yields 


$$
\Phi_{\omega \ell}^{-}(y) \simeq(1-y)^{ \pm \frac{i \tilde{\omega}}{2 \pi \bar{T}_{c}}}[1+\mathcal{O}(1-y)]
$$

where $\tilde{T}_{c}$ is the (dimensionless) cosmological horizon temperature defined below (2.3). Imposing outgoing boundary conditions at the cosmological horizon demands choosing the lower sign in (4.4). We will use a Chebyshev collocation scheme to numerically solve for $\left(\omega r_{c}, \Phi_{\omega \ell}^{-}\right)$(see [21] for a review on the subject). Hence, we want to perform a field redefinition that automatically enforces the above boundary conditions. This motivates the wave function redefinition

$$
\Phi_{\omega \ell}^{-}(y)=y^{-\frac{i \tilde{\omega}}{2 \pi \bar{T}_{+}}}\left(2-y^{2}\right)^{-\frac{i \tilde{\omega}}{4 \pi \bar{T}_{+}}}\left(1-y^{2}\right)^{-\frac{i \tilde{\omega}}{2 \pi \bar{T}_{c}}} \mathcal{Q}_{\omega \ell}(y),
$$

where, for our choice of boundary conditions, $\mathcal{Q}_{\omega \ell}(y)$ is a smooth function of $y$ with a regular Taylor series expansion at both $y=0$ and $y=1$. The boundary conditions for $\mathcal{Q}_{\omega \ell}(y)$ are then of the Neumann type, i.e., $\left.\partial_{y} \mathcal{Q}_{\omega \ell}(y)\right|_{y=0,1}=0$.

Consider now the eigenvalue problem II listed above. This time we have an extremal black hole, meaning that at the event horizon $f$ vanishes quadratically. It follows that, instead of (4.2), this time a Frobenius analysis about $y=0$ yields

$$
\Phi_{\omega \ell}^{-}(y) \simeq e^{ \pm i \tilde{\omega} \frac{\alpha}{y^{2}}} y^{ \pm i \tilde{\omega} \beta}[1+\mathcal{O}(y)]
$$

where

$$
\begin{aligned}
& \alpha \equiv \frac{y_{+}^{2}}{2(n-1)\left(1-y_{+}\right)} \frac{(n+1) y_{+}^{2 n}-2 n y_{+}^{n+1}+n-1}{4 n y_{+}^{n+1}-(n+1)\left(y_{+}^{2 n}+n y_{+}^{2}\right)+(n-1)^{2}}, \\
& \beta \equiv 2 n y_{+}\left[(n+1) y_{+}^{2 n}-2 n y_{+}^{n+1}+n-1\right] \frac{4(1-2 n) y_{+}^{n+1}+(n+1)\left[y_{+}^{2 n}+(3 n-2) y_{+}^{2}\right]-3(n-1)^{2}}{3(n-1)\left[4 n y_{+}^{n+1}-(n+1)\left(y_{+}^{2 n}+n y_{+}^{2}\right)+(n-1)^{2}\right]^{2}} .
\end{aligned}
$$

Requiring ingoing boundary conditions at the event horizon of the extremal RNdS black hole amounts to choose the upper sign in (4.6). For the extremal RNdS, a Taylor expansion about the cosmological horizon still yields (4.4) and choosing the lower sign in (4.4) still amounts to impose outgoing boundary conditions at the cosmological horizon, alike in the nonextremal case. Much like in the nonextremal case, since we use a pseudospectral collocation scheme, these boundary conditions are best implemented if we introduce the field redefinition

$$
\Phi_{\omega \ell}^{-}(y)=e^{\frac{i \tilde{\omega} \alpha}{y^{2}\left(2-y^{2}\right)}} y^{i \tilde{\omega} \beta}\left(1-y^{2}\right)^{-\frac{i \tilde{\omega}}{2 \pi \bar{T}_{c}}} \mathcal{Q}_{\omega \ell}(y),
$$

such that the above boundary conditions simply translate into Neumann conditions, namely, $\left.\partial_{y} \mathcal{Q}_{\omega \ell}(y)\right|_{y=0,1}=0$ for the smooth function $\mathcal{Q}_{\omega \ell}(y)$.

Finally, let us consider the nonlinear eigenvalue problem III listed above. In this case we want to find the eigenpair $\left(q / q_{\mathrm{ext}}, \Phi_{\omega \ell}^{-}\right)$that describes the onset of the instability in the nonextremal RNdS as we scan the $y_{+}$parameter. The boundary conditions for this problem are straightforward: a Frobenius analysis about $y=0(y=1)$ indicates that we have a term that diverges as $A \log y(a \log (1-y))$. We impose boundary conditions that eliminate these divergent terms: $A \equiv 0, a \equiv 0$ by taking pure Neumann boundary conditions for $\Phi_{0 \ell}^{-}(y)$.

\section{B. Numerical results}

The near-horizon analysis of the extremal RNdS black hole and associated Durkee-Reall criterion $[19,20]$ discussed in Sec. III suggests that near-extremal black holes should be unstable for $n \geq 4$. To confirm this is indeed the case, we first search for unstable modes directly in the extremal RNdS black hole using the numerical scheme II outlined in Sec. IVA. We find three main results:

(1) The gravitational instability in the RNdS black hole is present when the spacetime dimension satisfies $n \geq 4(d \geq 6)$ and $\ell=2$. In particular, it is present for $d=6(n=4)$, as predicted by the near-horizon criterion, which is a result that was not established in previous literature. This is explicitly shown for $n=4$ in the top-left panel of Fig. 2 and for $n \geq 5$ in the bottom panel of Fig. 2. These plots display the imaginary part of the dimensionless frequency, $\operatorname{Im}\left(\omega r_{c}\right)$, as a function of $y_{+}=r_{+} / r_{c}$ (the real part of the frequency of the unstable modes vanishes).

Because the $n=4$ case has such a small instability timescale, it is important to do convergence tests of our solutions to validate them, i.e., to guarantee that they are indeed physical and not within the error bar. To get our results, we have used a numerical radial grid with $N$ points. For reference, for $n=4$ we had to use $N=12000$ while, for $n>4$, typically we just had to use $N=600$. This fact and the tiny timescales in the $n=4$ case justify why we did not attempt to extend the instability curve of the top-left plot of Fig. 2 to lower either higher values of $y_{+}$. The convergence of the quantity $\Gamma \equiv \operatorname{Im}\left(\omega r_{c}\right)$ can be measured using the function

$$
R_{\Gamma}(N)=100\left|1-\frac{\Gamma_{N}}{\Gamma_{N+1000}}\right|,
$$



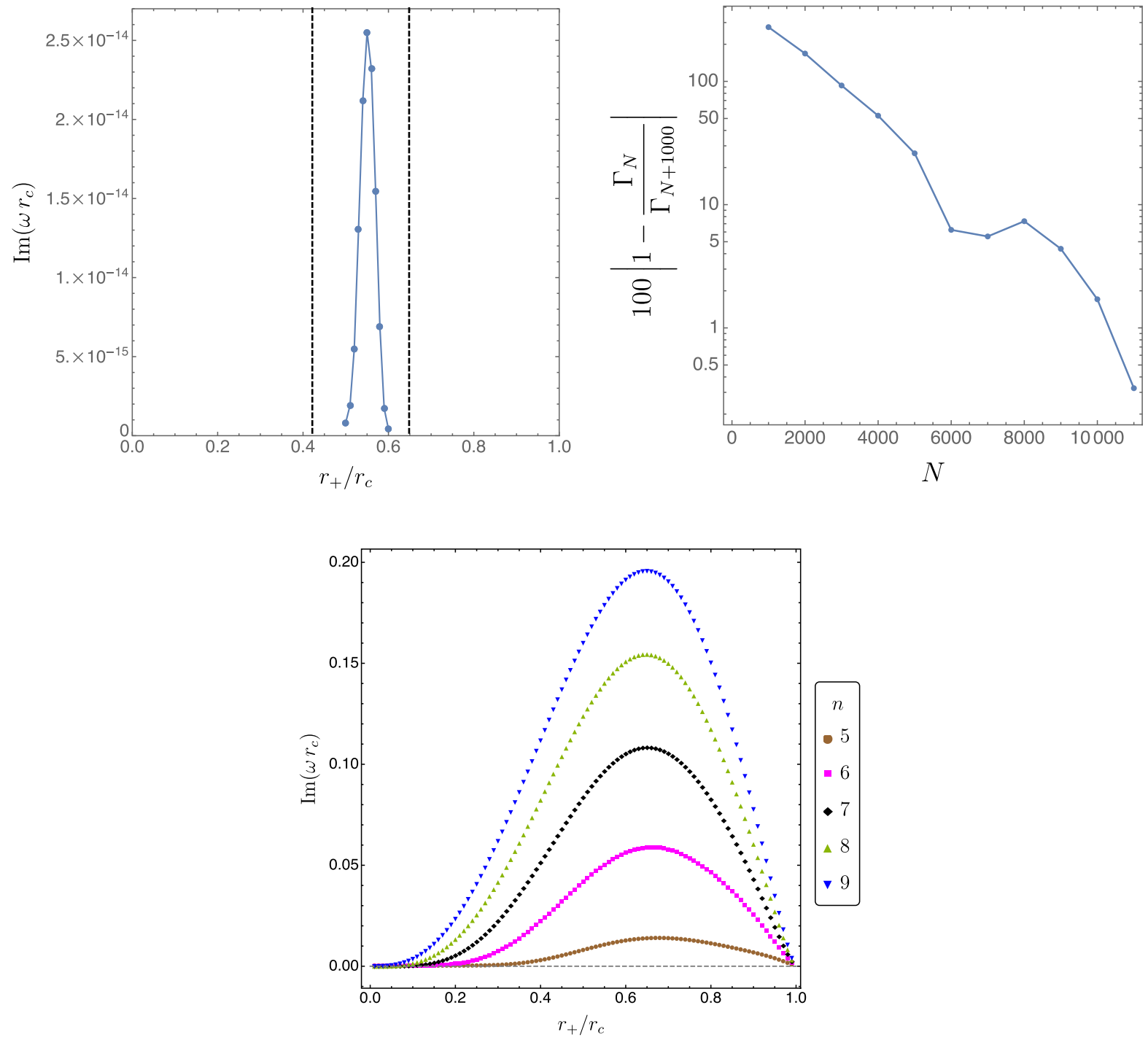

FIG. 2. Instability timescale at extremality, $q=q_{\text {ext }}$ as a function of $r_{+} / r_{c}$ (the real part of the frequency of these unstable modes vanishes). Top panel: The $d=6(n=4)$ case. In the left plot, the Durkee-Reall criterion is satisfied in between the two black dashed vertical lines and the blue dots describe the unstable modes we found numerically in the region where the instability is the strongest. The top-right panel displays the convergence of $\Gamma \equiv \operatorname{Im}\left(\omega r_{c}\right)$ as a function of the number of grid points $N$ we have done for the first solution, with $r_{+} / r_{c}=0.5$, of the left panel curve. Bottom panel: The distinct curves describe different spacetime dimensions, $d=n+2 \geq 5$ (see legend). The instability is stronger for higher $n$ but, at extremality, it is always present for any value of $r_{+} / r_{c}$ in the allowed range ]0, 1 [.

which vanishes for large $N$ for any converging numerical method. Since we are using pseudospectral collocation, $R_{\Gamma}$ should decrease exponentially in $N$ if the solution is sufficiently smooth. The solution that was hardest to find was the one described by the first point, with $r_{+} / r_{c}=0.5$, on the top-left panel of Fig. 2. We therefore perform a convergence test for this solution in the top-right panel of Fig. 2. This convergence test shows that our analysis has the exponential convergence (notice the logarithmic scale) expected when pseudospectral collocation methods are used and also shows that the error in our computation is well below the given timescale.
(2) At extremality, $q / q_{\mathrm{ext}}=1$, the instability is present for any value of $y_{+}=r_{+} / r_{c}$, i.e., for $y_{+} \in[0,1]$ (when $n \geq 4$ and $\ell=2$ ) with $\operatorname{Im}\left(\omega r_{c}\right) \rightarrow 0$ as $y \rightarrow 0$ or $y \rightarrow 1$ and attaining a maximum in between. This is explicitly shown for $n \geq 5$ in the bottom panel of Fig. 2. For $n=4$ it is computationally much harder to generate the associated numerical data but we believe this case should behave much like the $n \geq 5$ cases. Note that in previous literature [2,4], the presence of the instability was established only for large values of $r_{+} / r_{c}$ (and $n \geq 5$ ) and later it was (incorrectly) claimed that the instability is present only above a critical value of $y_{+}$[3]. 

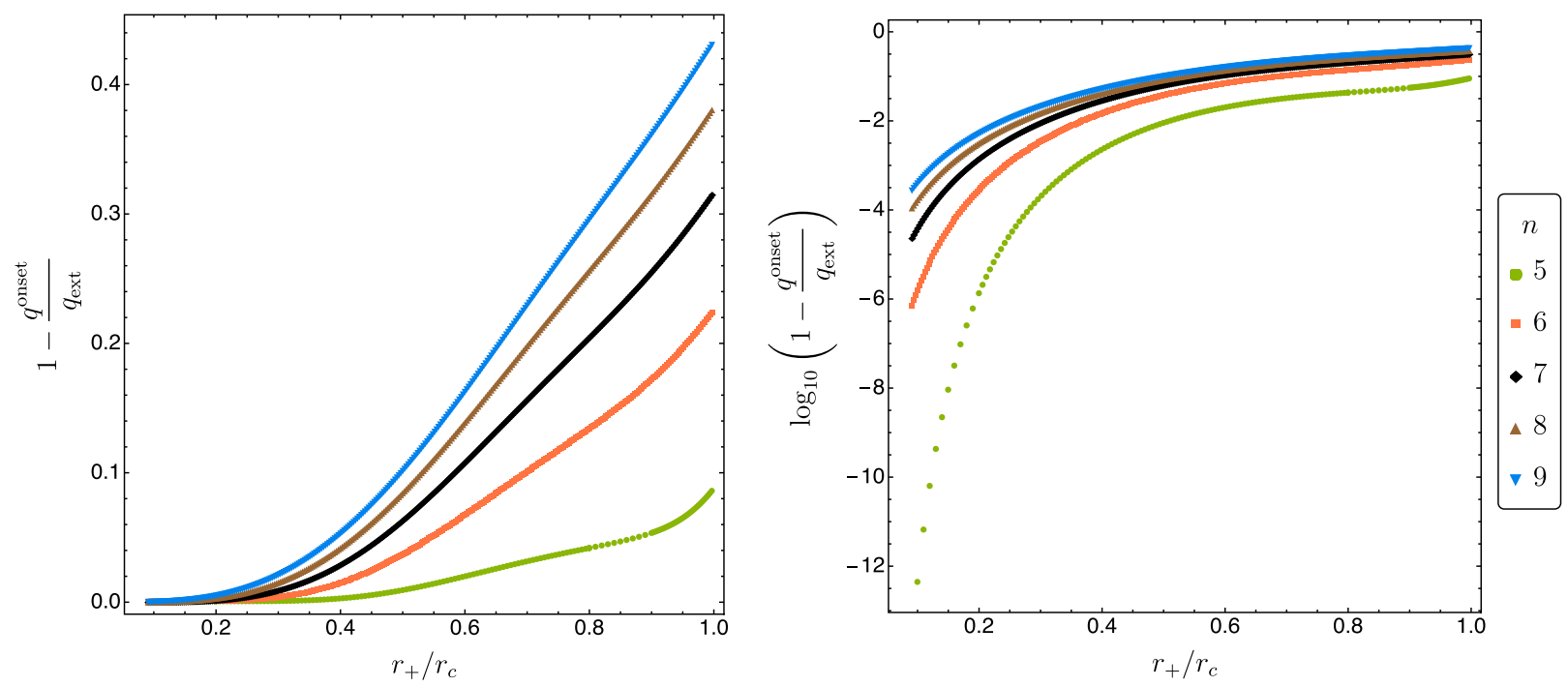

FIG. 3. Instability onset. For given $r_{+} / r_{c}$, RNdS black holes with $q>q^{\text {onset }}$ are unstable. The right panel is simply a log plot of the left panel to better see what happens for small $y_{+}$.

(3) The near horizon Durkee-Reall criterion $[19,20]$ discussed in Sec. III provides a sufficient but not necessary condition for the instability. That is to say, our numerical results show that the system is indeed always unstable whenever the $\mathrm{AdS}_{2} \mathrm{BF}$ bound is violated. But the instability also extends to values of the parameter space where the near-horizon criterion does not signal an instability. This is best seen comparing the analytical near-horizon predictions of Fig. 1 with the actual numerical results of Fig. 2. Recall that both these plots are for $\ell=2$ and $q=q_{\text {ext }}$. Typically, the near-horizon analysis of Fig. 1 predicts instability for a finite range of $y_{+}$. However, we find that, at extremality, the system is unstable in the full range $y_{+} \in[0,1]$. This is certainly the case for $n \geq 5$ (see bottom panel of Fig. 2) and should also be true for $n=4$.

Having established that all, $0 \leq y_{+} \leq 1$, extremal RNdS black holes are unstable for $n \geq 4$ we might then ask how far away from extremality does the instability extend into. To address this question we first search directly for the onset of the instability using the numerical scheme III outlined in Sec. IVA. This critical charge $q^{\text {onset }}$ above which the RNdS solution is unstable is shown in Fig. 3 for $n=5,6,7,8,9$ and $\ell=2$. The left panel shows $1-q^{\text {onset }} / q_{\text {ext }}$ as a function of $y_{+}$while the right panel shows the logarithmic plot of the same quantity to zoom the details of the small $y_{+}$region. We see that for a given dimension $n, q^{\text {onset }} / q_{\text {ext }}$ decreases as $y_{+}$grows from 0 into 1: the instability extends further away from extremality for high $y_{+}$. On the other hand, for a given $y_{+}$we see that increasing the dimension $n$ favors the instability in the sense that $q^{\text {onset }} / q_{\text {ext }}$ becomes smaller as $n$ grows.

To have a broader perspective of the properties of the instability, next we search directly for the instability timescale in the nonextremal RNdS black hole. For this discussion we fix the dimension to be $n=5$ and the associated data is shown in Fig. 4 (for other dimensions the plot is qualitatively similar). Recall that nonextremal RNdS black holes are parametrized by $q / q_{\mathrm{ext}}$ and $r_{+} / r_{c}$ and these are the two horizontal axes of Fig. 4. On the other hand the vertical axis is the imaginary part of the dimensionless frequency, $\operatorname{Im}\left(\omega r_{c}\right)$ (the real part of the frequency of the

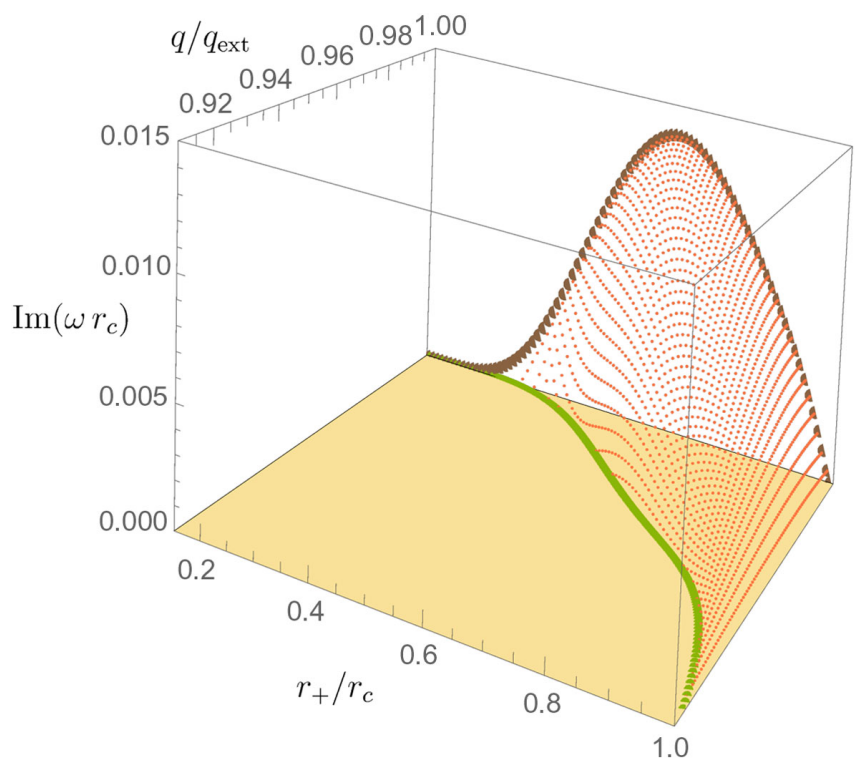

FIG. 4. Instability timescale for $n=5$ : imaginary part of the frequency $\operatorname{Im}\left(\omega r_{c}\right)$ as a function of $r_{+} / r_{c}$ and $q / q_{\text {ext }}$ [only shown the parameter space region where the instability is present, i.e., that has $\left.\operatorname{Im}\left(\omega r_{c}\right) \geq 0\right]$. The brown dots in the plane $q / q_{\text {ext }}=1$ represent instability data collected independently using a numerical code at extremality. The green dots with $\operatorname{Im}\left(\omega r_{c}\right)=0$ represent data collected independently using a numerical code for the onset of the instability. 
unstable modes vanishes). In this three-dimensional plot we also show the extremal (brown) curve already displayed in Fig. 2 and the onset (green) curve already shown in Fig. 3 (for $n=5$ ). The fact that the two-dimensional surface describing the regime where RNdS is unstable ends on the extremal and onset curves obtained using independent numerical codes provides a nontrivial check of our numerical results. This plot reveals the following properties (some were already discussed above):

(i) At extremality, $q / q_{\text {ext }}=1$, the instability is present in the whole range $0 \leq y_{+} \leq 1$.

(ii) As we move away from extremality, we see that for a given (large) charge, namely, in the range $0.9128 \lesssim$ $q / q_{\text {ext }} \leq 1$ (when $n=5$ ) the system is unstable only for $y_{+}$above a (nonvanishing) critical value. For smaller charges $q$ the system is stable. In equivalent words, for a given $y_{+}$, RNdS black holes are unstable if their charge is above $q^{\text {onset }}$, with $q^{\text {onset }}$ approaching $q_{\text {ext }}$ as $y_{+} \rightarrow 0$.

(iii) The maximum strength of the instability is attained for black holes that are close to extremality, but not at extremality. This is better seen in Fig. 5 where we show the instability timescale for three families of $\mathrm{RNdS}$ solutions at constant $y_{+}$(namely, $y_{+}=0.70,0.95$, 0.99 ) as a function of the dimensionless charge ratio $q / q_{\text {ext }}$. We see that typically $\operatorname{Im}\left(\omega r_{c}\right)$ grows as $q / q_{\text {ext }}$ increases but its maximum occurs slightly before one reaches the extremal configuration $q / q_{\text {ext }}=1$.

Up until now we have discussed only modes with $\ell=2$. This is because for each dimension $n \geq 4$ this is either

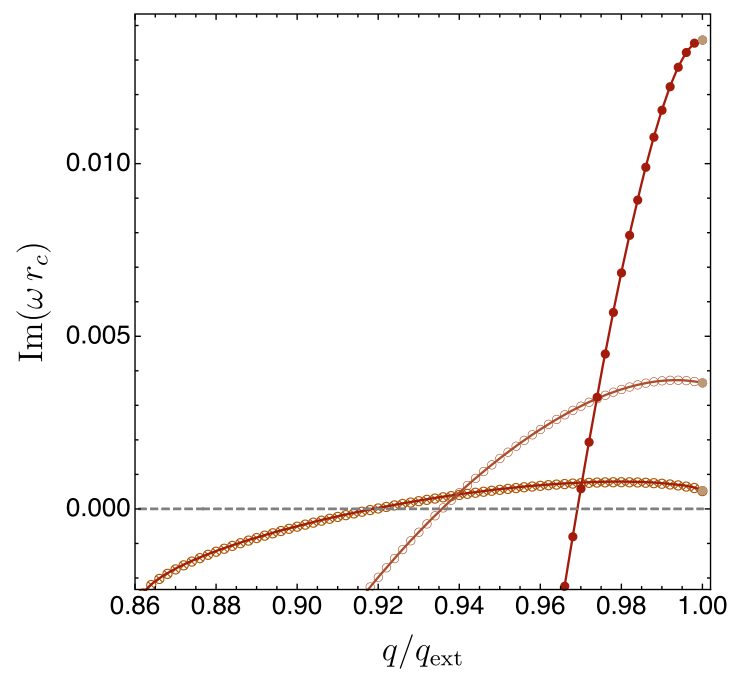

FIG. 5. Frequency spectrum (imaginary part) for RNdS black hole families with $y_{+}=0.70$ (Black circle), $y_{+}=0.95$ (White circle), and $y_{+}=0.99$ (N-ary Circled Times) and $n=5, \ell=2$. The brown disks with $q / q_{\text {ext }}=1$ were obtained using the independent code II for the extremal solution. The onset of the instability [where $\operatorname{Im}\left(\omega r_{c}\right)=0$ ] occurs at the critical values $\left\{y_{+}, q / q_{\text {ext }}\right\}$ also obtained using the independent numerical code III used to generate the onset curve of Fig. 3. (i) the only harmonic for which the instability is present or (ii) the harmonic mode where the instability is stronger. But we can also discuss briefly other harmonics. As discussed in Sec. III, the near-horizon analysis shows that as $\ell$ increases it becomes harder to get negative $\left(\mu^{2}-\mu_{\mathrm{BF}}^{2}\right) L_{2}^{2}$. For example, in $n=4$ or $n=5$ dimensions, for $\ell \geq 3$ the $\mathrm{AdS}_{2} \mathrm{BF}$ bound is no longer violated for any $y_{+}$. As another example, for $n=6,7$ the harmonics $\ell=2,3$ generate a violation of the $\mathrm{AdS}_{2} \mathrm{BF}$ bound but this is no longer the case for $\ell \geq 4$. As a final example, for $n=8,9$ the harmonics $\ell=2,3,4$ generate a violation of the $\mathrm{AdS}_{2}$ $\mathrm{BF}$ bound but this is no longer the case for $\ell \geq 5$. The full numerical analysis confirms these analytical predictions to be correct. In particular, the numerical analysis also concludes that when the instability is present for more than the $\ell=2$ harmonic, modes with lower $\ell$ are more unstable. To illustrate this, in Fig. 6 we take an extremal $\mathrm{RNdS} \mathrm{BH}$ in $n=9$ and compare the instability timescale of $\ell=2$ and $\ell=3$ modes. We see that the $\ell=3$ instability timescale is typically 2 orders of magnitude smaller than the $\ell=2$ timescale. We have also explicitly checked that for $n=9$ the $\ell=4$ harmonic (but not higher $\ell$ 's) is also unstable but with a strength that is $\sim 5$ orders of magnitude smaller than the $\ell=2$ instability. For example, for $y_{+}=1 / 2$ the timescale of three harmonics are:

$$
\begin{aligned}
& \ell=2: \omega r_{c} \simeq 1.60 \times 10^{-1} i, \\
& \ell=3: \omega r_{c} \simeq 9.78 \times 10^{-3} i, \\
& \ell=4: \omega r_{c} \simeq 1.37 \times 10^{-6} i .
\end{aligned}
$$

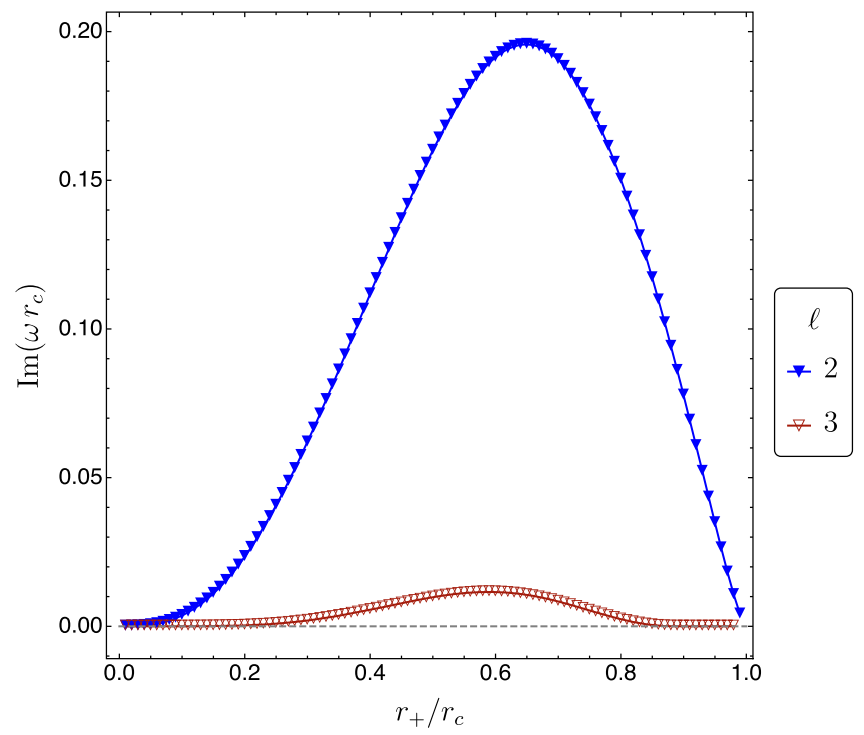

FIG. 6. Instability timescale at extremality, $q=q_{\text {ext }}$, for $n=9$ as a function of $r_{+} / r_{c}$ for the $\ell=2$ (blue "Black Down-pointing Triangle") and $\ell=3$ (red "White Down-pointing Triangle") harmonics. The $\ell=2$ curve was already shown (with same color/ shape code) in Fig. 2. 

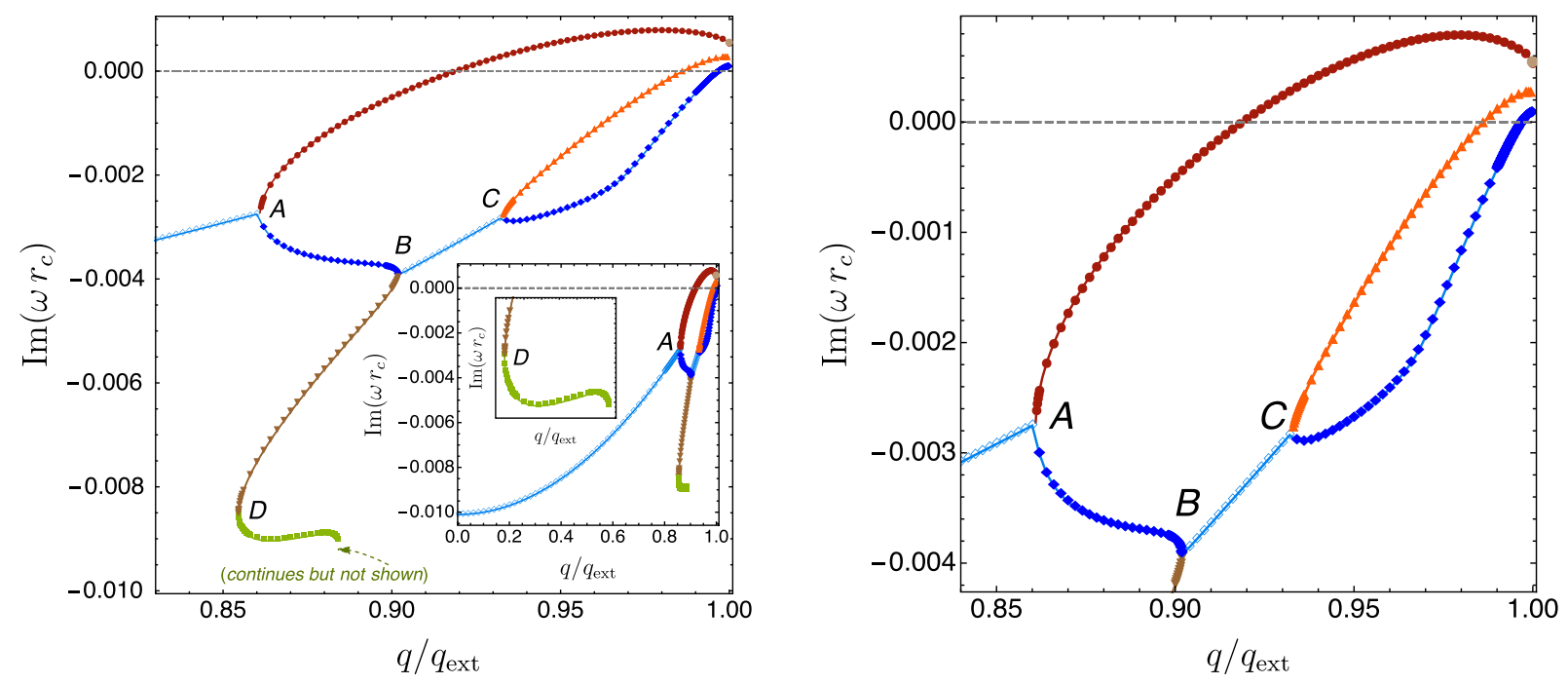

FIG. 7. Frequency spectrum (imaginary part) for $y_{+}=0.99$ and $n=5, \ell=2$ with zooms in the relevant regions where instability is present.

So far we have focused our attention on describing key properties of the instability present in RNdS black holes and confirming that we can understand its origin as being due to the violation of a relevant $\mathrm{AdS}_{2} \mathrm{BF}$ bound discussed in Sec. III. The relevant modes become unstable close to extremality.

Next, we give a broader view of the properties of these perturbations and follow the unstable modes as we move away from extremality all the way down to the Schwarzschild-dS black hole with $q=0$. This analysis turns out to reveal an interesting quasinormal mode structure with properties that, to the best of our knowledge, no other known black hole quasinormal mode spectrum exhibits. In our scan of solutions we have found three main regimes that are distinct from each other and thus deserve a separate discussion. Illustrative examples of each of these three cases are the RNdS black holes with $y_{+}=0.70$, $y_{+}=0.95$, and $y_{+}=0.99$ (and $n=5, \ell=2$ ). The main instability properties near extremality of these three cases were already discussed in Fig. 5. Next we extend the analysis of these modes all the way towards $q=0$, i.e., well away from the region where the solutions are unstable and we also discuss "secondary" (less unstable or stable) modes that are nevertheless relevant to have a good overview of the quasinormal mode structure. The key properties of these three families can be summarized as follows:

(1) The case $y_{+}=0.99$ represents what typically happens for RNdS black holes that have very large $y_{+}$ (i.e., close to unit). The relevant frequency spectrum for this case is displayed in Fig. 7 (imaginary part) and Fig. 8 (real part of the frequency). In Fig. 7 we identify the dark red disk $(\bullet)$ curve that describes the (most) unstable mode that was already identified in Fig. 5 with red $\otimes$ (and in Fig. 4) and that has $\operatorname{Re}\left(\omega r_{c}\right)=0$. In particular, the brown disk with $q / q_{\mathrm{ext}}=1$ in this curve describes the extremal solution also identified in Fig. 2. We see that this curve has a maximum close to extremality (as discussed previously) and decreasing $q / q_{\text {ext }}$ we find that the mode first becomes stable at $q / q_{\text {ext }} \sim 0.92$ (consistent with Fig. 3) and then reaches a bifurcation point $A$ at $q / q_{\text {ext }} \sim 0.861$ where it joins the light blue lozenge $(\diamond)$ and blue diamond $(\diamond)$ curves. As seen in the inset plot of the left panel, the light blue $\diamond$ branch extends all the way from $A$ down to $q=0$. As shown in the companion, Fig. 8, the real part of the frequency of this branch is always nonvanishing $\left[\operatorname{Re}\left(\omega r_{c}\right) \neq 0\right]$ and it decreases monotonically as $q$ increases from zero towards the bifurcation point $A$ with $q / q_{\text {ext }} \sim 0.861$. At this point $A$, the light blue $\diamond$ branch bifurcates into the dark red - curve (upper branch) already discussed above and into the blue $\downarrow$ curve (lower branch). These two branches $(\bullet$ and $\$$ ) both have $\operatorname{Re}\left(\omega r_{c}\right)=0$. That is to say, a complex eigenvalue $^{3}$ bifurcates into two branches that have purely imaginary eigenvalues. Now let us follow the blue diamond branch. We see that it extends from point $A$ with $q / q_{\text {ext }} \sim 0.861$ up to point $B$ with $q / q_{\text {ext }} \sim 0.902$. At this point $B$ it merges with the brown inverted triangle $\boldsymbol{\nabla}$ curve (that also approaches point $B$ but coming from point $D$ ) and the solution now extends for higher $q / q_{\text {ext }}$ along the light blue $\diamond$ branch that again has $\operatorname{Re}\left(\omega r_{c}\right) \neq 0$ (see the half-circle $B C$ section of Fig. 8) up to point $C$ with $q / q_{\text {ext }} \sim 0.934$. At this point $C$ we have again a new bifurcation into two branches: the upper branch

\footnotetext{
${ }^{3}$ Since RNdS is a static background with Killing vector $\partial_{t}$, if $\omega=\omega_{R}+i \omega_{I}$ is an eigenvalue then $-\omega^{*}=-\omega_{R}+i \omega_{I}$ is also an eigenvalue of the frequency spectrum.
} 

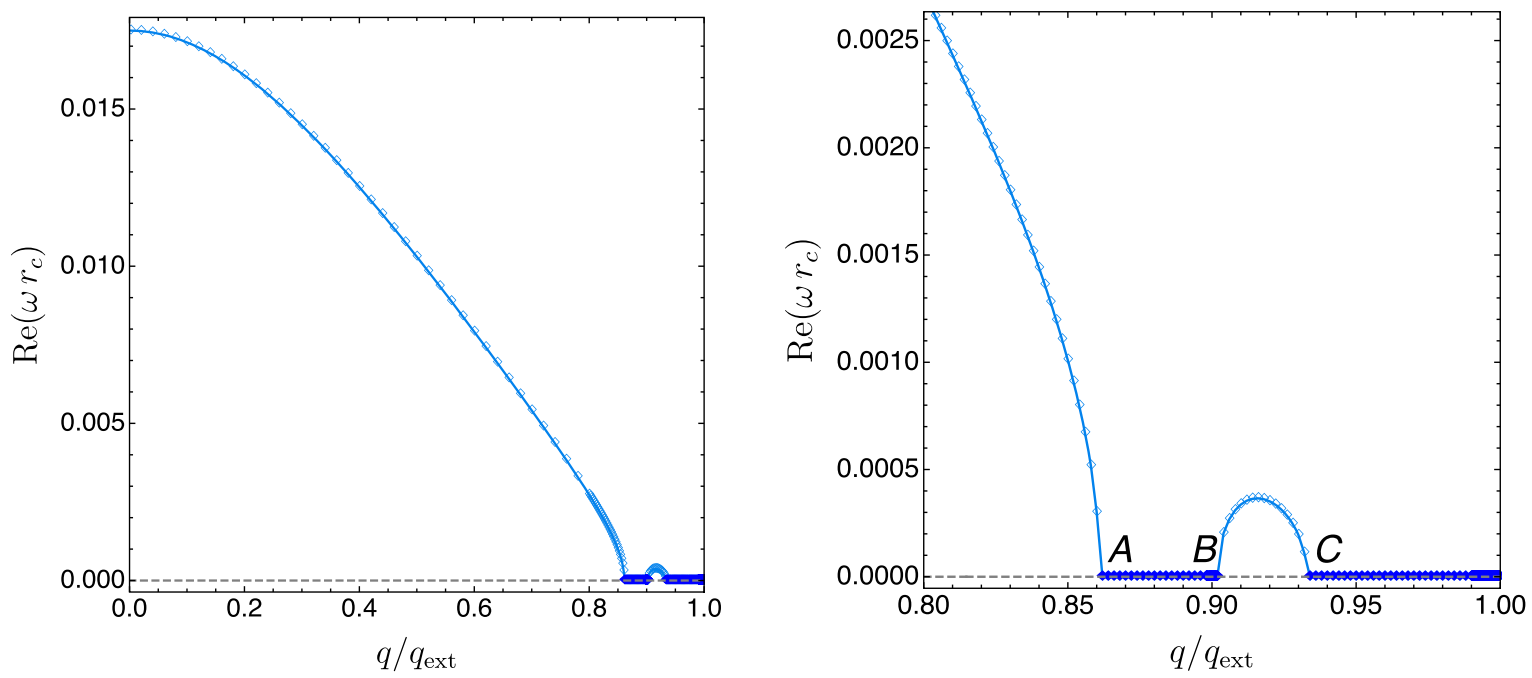

FIG. 8. Frequency spectrum (real part) for $y_{+}=0.99$ and $n=5, \ell=2$. The right panel zooms in the near-extremal region where the instability is present. The green, red, dark red and orange sections of Fig. 7 are not shown: they all have $\operatorname{Re}\left(\omega r_{c}\right)=0$.
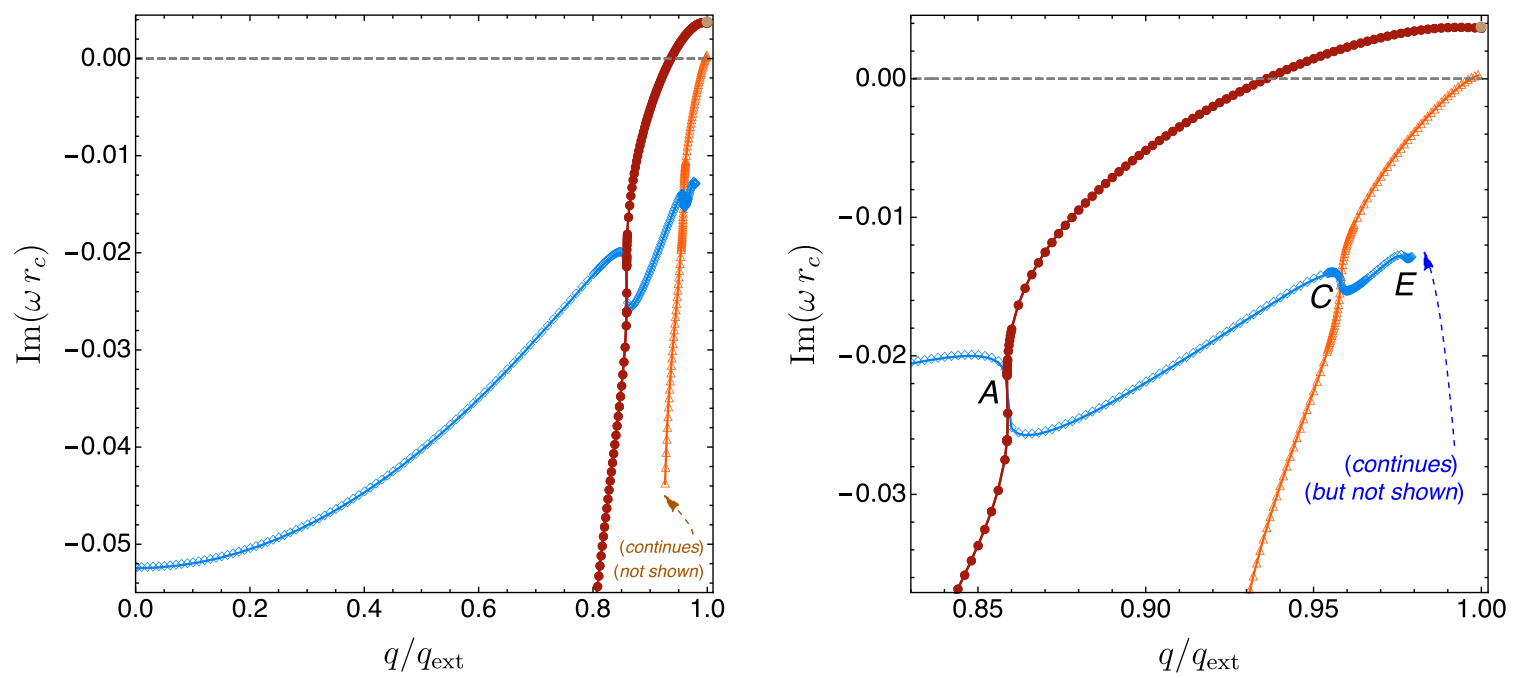

FIG. 9. Frequency spectrum (imaginary part) for $y_{+}=0.95$ and $n=5, \ell=2$ with zooms in the relevant regions where instability is present.

with orange triangles $(\mathbf{\Lambda})$ and the lower branch with blue diamonds $(\checkmark)$. Both these branches then extend independently towards extremality $\left(q / q_{\text {ext }}=1\right)$ in such a way that $\operatorname{Im}\left(\omega r_{c}\right)$ grows monotonically from negative into positive values [moreover $\operatorname{Re}\left(\omega r_{c}\right)=0$ for both branches]. This is best seen in the zoomed right panel of Fig. 7. For completeness we should also mention the turning point $D$ seen in the left panel of Fig. 7 where the brown inverted triangle $\boldsymbol{\nabla}$ curve continues as the green square (๘). Both curves have $\operatorname{Re}\left(\omega r_{c}\right)=0$. The green square curve extends away from $D$ to higher $q / q_{\text {ext }}$ where it has a new turning point but we do not analyze/discuss this further.
Although we have not done an exhaustive scan of the parameter space, we have collected enough evidence that the frequency spectrum for RNdS black holes with $y_{+}$close to 1 has a bifurcation/ merger structure qualitatively similar to the one shown in Fig. 7 and Fig. 8 (some of this evidence comes from Figs. 12-13, 14-15, and 16-17 to be discussed later). It is also important to point out that a bifurcation structure similar to the one seen around point $A$ of Figs. 7-8 also emerges from a large $d$ analysis of the instability: see Fig. 1 of [6]. However, at least for finite values of the dimension, the system seems to have a more complicated bifurcation structure than the one reported in [6]: there are 

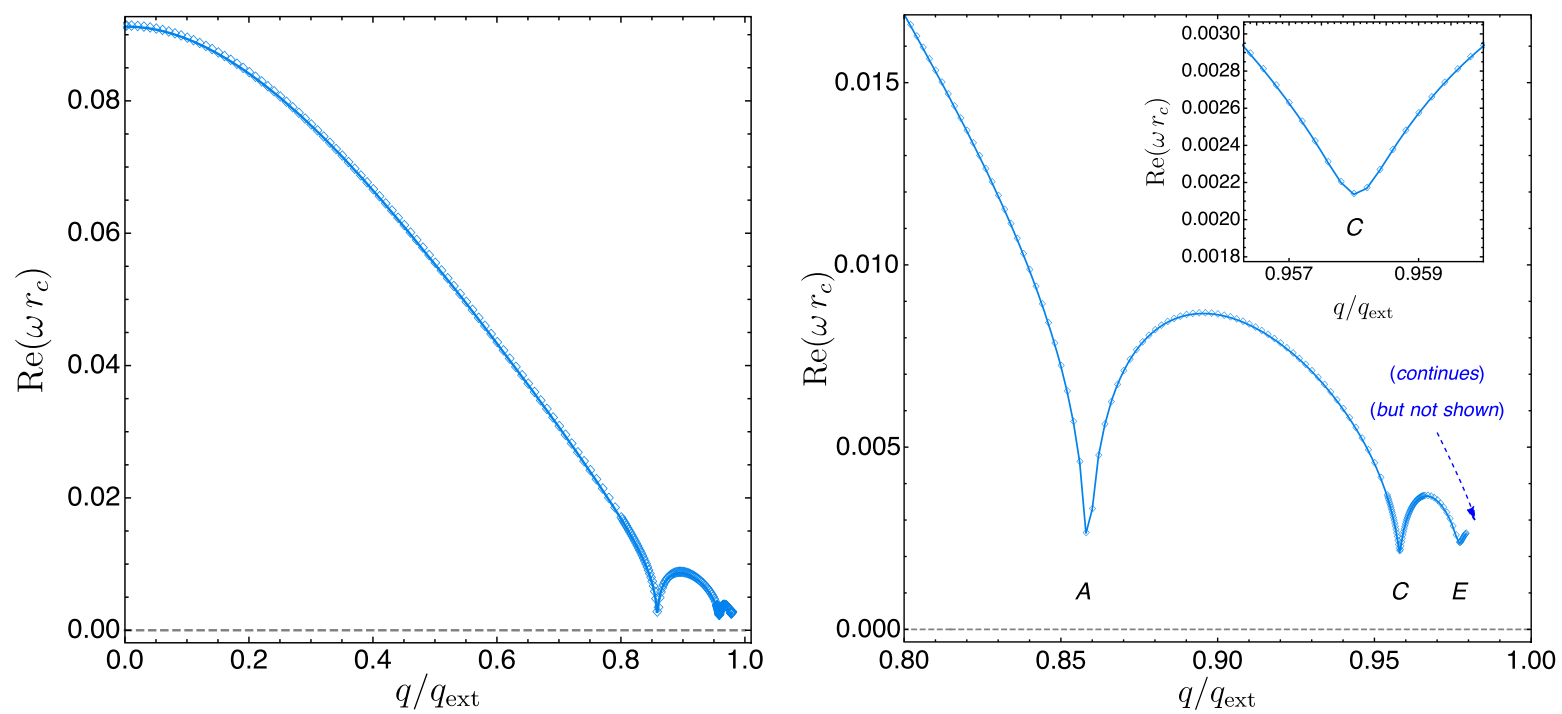

FIG. 10. Frequency spectrum (real part) for $y_{+}=0.95$ and $n=5, \ell=2$ with zooms in the relevant regions where instability is present. The dark red and orange sections of Fig. 9 are not shown: they both have $\operatorname{Re}\left(\omega r_{c}\right)=0$.
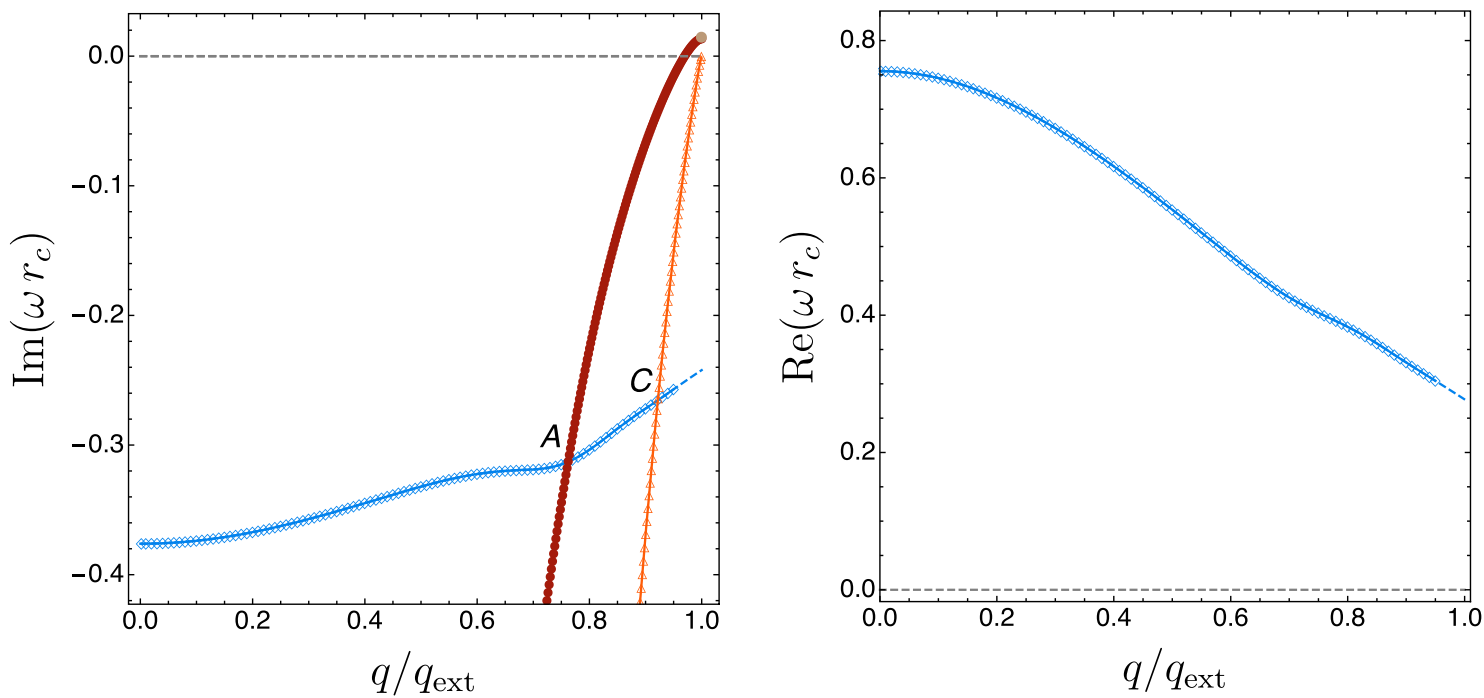

FIG. 11. Frequency spectrum (left panel: imaginary part, right panel: real part) for $y_{+}=0.70$ and $n=5$, $\ell=2$ with zooms in the relevant regions where instability is present. Right panel: Real part of the frequency. The dark red "Black circle" and orange "up-pointing triangle" branches of the left panel have $\operatorname{Re}\left(\omega r_{c}\right)=0$ and are not shown.

further bifurcations/mergers like $B$ and $C$ in Figs. 7-8.

(2) Next we consider the case $y_{+}=0.95$ which represents RNdS that have moderately large $y_{+}$that is, however, not large enough to be close to unit. The relevant frequency spectrum for this case is displayed in Fig. 9 (imaginary part) and Fig. 10 (real part of the frequency). This case is distinguished from the previous case mainly because the bifurcations/mergers (like $A$ and $C$ of Fig. 7) cease to exist. Instead they are replaced by crossovers of quasinormal mode branches. Take the left panels of
Figs. 9 and 10. As before, the light blue lozenge $(\diamond)$ curve starts at $q=0$ and has $\operatorname{Re}\left(\omega r_{c}\right) \neq 0$. This curve extends all the way to extremality (although it becomes very difficult to compute the properties of this curve for $q / q_{\text {ext }}$ above 0.98 ) with an intricate structure best seen in the zoom plots of the right panels of Figs. 9 and 10. Indeed, in the $\operatorname{Im}\left(\omega r_{c}\right)$ plot the light blue lozenge $(\diamond)$ curve has some zigzagged regions that correspond to minima cusp points in the $\operatorname{Re}\left(\omega r_{c}\right)$ plot: these are regions $A, C, E, \cdots$ in Figs. 9 and 10 . These regions coincide with the points where the dark red disk $(\bullet)$ and orange triangle $(\triangle)$ curves 

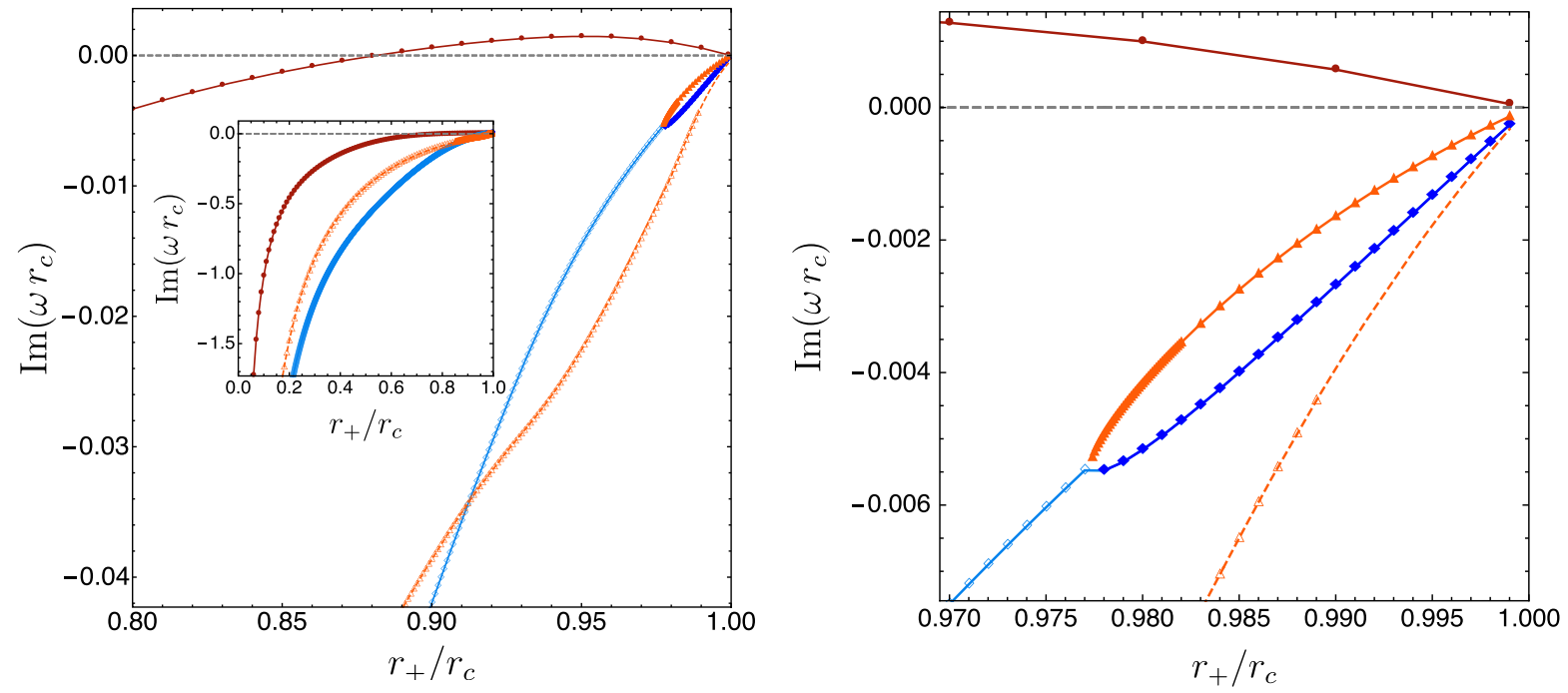

FIG. 12. Left and right panels: Frequency spectrum (imaginary part) for $q / q_{\text {ext }}=0.95$ and $n=5, \ell=2$ with zooms in relevant regions. We use the same shape/color code (Black circle, White daimond, up-pointing black filled traingle, Black filled daimond, up-pointing white triangle) used in Figs 7-8, 9-10, and 11.

[that have both $\operatorname{Re}\left(\omega r_{c}\right)=0$ ] cross over the light blue lozenge $(\diamond)$ curve (note that the red disk curve describing the most unstable mode is precisely the one already displayed in Fig. 5 as a dark-red ०). It is important to emphasize that $A$ and $C$ describe crossover not intersection points [since the eigenfunctions of the branches that intersect are distinct and points $A, C$, and $E$ in the light blue $\diamond$ curve have $\operatorname{Re}\left(\omega r_{c}\right) \neq 0$ unlike in the $\bullet$ and $\triangle$ curves $)$. Comparing with the situation of Fig. 7 and Fig. 8 we can say that the bifurcation/merger points $A$ and $C$ of Fig. 7 become crossover points in Fig. 9. Further note that, although not shown in Fig. 9, there should exist at least another family of modes to the right of the orange $\triangle$ curve and "parallel" to it that should crossover the $\diamond$ curve in region $E$. Finally, we should pointed out that the orange empty $(\triangle)$ triangle modes of Fig. 9 are not the extension of the orange filled triangle $(\boldsymbol{\Lambda})$ modes of Fig. 7: this will become clear later when we discuss the curves $\boldsymbol{\Delta}$ and $\Delta$ of Fig. 12.

(3) Finally, we consider the case with $y_{+}=0.70$ which is a representative case of what happens with $\mathrm{RNdS}$ black holes that have intermediate and small values of $y_{+}$. The relevant frequency spectrum for this case is displayed in Fig. 11 (imaginary part in left panel and real part in the right panel). This case distinguishes from the previous case because the structure of the spectrum is now very simple: the zigzag regions on the $\operatorname{Im}\left(\omega r_{c}\right)$ plots of Fig. 9, i.e., the cusps in the $\operatorname{Re}\left(\omega r_{c}\right)$ plots of Fig. 10, have now flattened out completely and we have very simple crossovers $A$ and $C$ between the light blue lozenge $(\diamond)$ [which has $\operatorname{Re}\left(\omega r_{c}\right) \neq 0$ ] and, respectively, the red disk (•) curve that is unstable (already shown in Fig. 5 as a dark-red $\bullet$ ) and the orange triangle $(\triangle)$ curve.

As observed before, the RNdS family of black holes spans a two-dimensional parameter space that we are taking to be the dimensionless ratios $y_{+}=r_{+} / r_{c}$ and $q / q_{\mathrm{ext}}$. In the sequence of Figs. 7-8, 9-10, and 11 we have always fixed $y_{+}$and analyzed how the frequency spectrum changes as the charge of the RNdS black hole changes. Next, to have a three-dimensional perspective of the system, we complement this analysis: we choose some relevant RNdS solutions with a fixed charge $q / q_{\text {ext }}$ and discuss how their

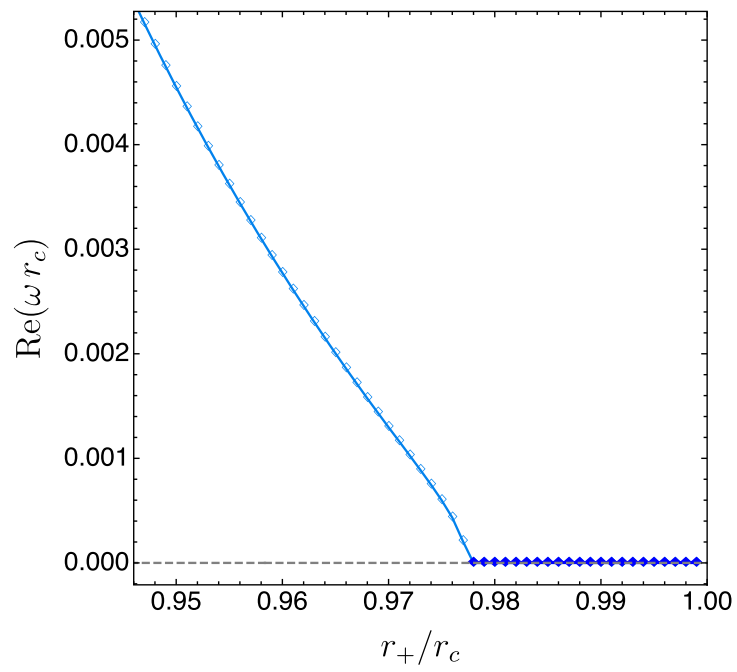

FIG. 13. Frequency spectrum (real part) for $q / q_{\mathrm{ext}}=0.95$ and $n=5, \ell=2$ with zooms in relevant regions. The dark red "Black circle" and orange "up-pointing black filled traingle, up-pointing white triangle" branches of Fig. 12 are not shown because they simply have $\operatorname{Re}\left(\omega r_{c}\right)=0$. 

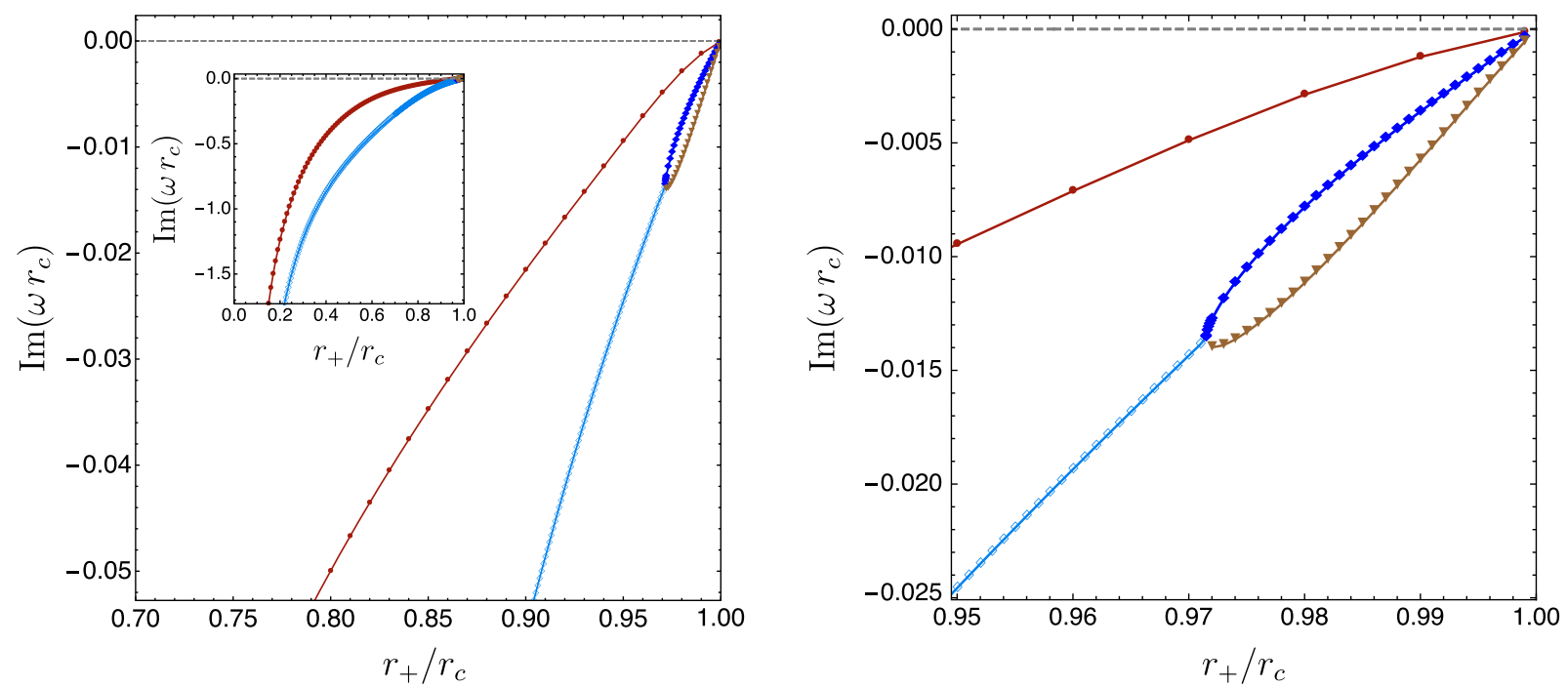

FIG. 14. Left and right panels: Frequency spectrum (imaginary part) for $q / q_{\text {ext }}=0.88$ and $n=5, \ell=2$ with zooms in relevant regions. We use the same shape/color code (Black circle, White daimond, Black filled daimond, Down-pointing black triangle) used in Figs 7-8, 9-10, and 11 .

frequency changes as $y_{+}$changes (we take again $n=5$ and $\ell=2$ ). Particularly illustrative cases that further help revealing the properties of the system are $q / q_{\text {ext }}=0.95$, $q / q_{\mathrm{ext}}=0.88$, and $q / q_{\mathrm{ext}}=0.85$. The analysis of these three cases unveils the following properties:

(1) We start with a RNdS black hole with $q / q_{\mathrm{ext}}=0.95$. In Fig. 12 (imaginary part) and Fig. 13 (real part) we show how the frequency $\omega r_{c}$ changes as we vary $y_{+}$. The curve on the top of Fig. 12 with dark red disks $(\bullet)$ is the branch that is unstable for large $y_{+}$. It confirms, as already seen in Fig. 3, that RNdS with $q / q_{\mathrm{ext}}=0.95$ become unstable for $y_{+} \gtrsim 0.881$ (see left panel of Fig. 12). Additionally, out of an infinite family of modes with $\operatorname{Im}\left(\omega r_{c}\right)<0$ for any $y_{+}$, we further show the two families of modes that have the smallest $\left|\operatorname{Im}\left(\omega r_{c}\right)\right|$. These are (i) the light blue $\diamond$ branch [that has $\operatorname{Re}\left(\omega r_{c}\right) \neq 0$; see Fig. 13] that bifurcates at $q / q_{\mathrm{ext}} \sim 0.977$ into two branches with $\operatorname{Re}\left(\omega r_{c}\right)=0$, namely, the orange triangle ( $\left.\mathbf{\Lambda}\right)$ branch and the solid blue diamond $(\checkmark)$ branch and (ii) the orange empty triangle $(\triangle)$ branch. A zoom in of the region close to $y_{+}=1$ is displayed in the right panel of Fig. 12. This plot at constant $q / q_{\mathrm{ext}}=0.95$ complements well those plots at constant $y_{+}$shown previously (namely, Figs. 7, 9, and 11). For example, in the right panel of Fig. 12, we identify the upper three points $\bullet, \mathbf{\Delta}, \boldsymbol{v}$ with $y_{+}=0.99$. These three points are the three points with the same shape/color code $\bullet, \boldsymbol{\Delta}, \diamond$ with $q / q_{\text {ext }}=0.95$ of Fig. 7 (which describes RNdS solutions with $y_{+}=0.99$ ). As another example, in the left panel of Fig. 12, we identify the three points $\bullet, \triangle, \triangle$ with $y_{+}=0.95$ that are the three points $\bullet, \Delta$ with $q / q_{\text {ext }}=0.95$ of Fig. 9 (which describes $\mathrm{RNdS}$ solutions with $\left.y_{+}=0.95\right)$. This is a good moment to pause and note, as observed in the end of the discussion of Fig. 9, that the orange $\boldsymbol{\Delta}$ (first introduced in Fig. 7) and $\Delta$ modes (first introduced in Fig. 9) are indeed distinct. As a final example, in the left panel of Fig. 12, we further identify the three points $\bullet, \triangle, \diamond$ with $y_{+}=0.70$ that are the three points $\bullet, \triangle, \checkmark$ with $q / q_{\mathrm{ext}}=0.95$ of Fig. 11 (which describes RNdS solutions with $y_{+}=0.70$ ).

(2) Next consider RNdS black holes with $q / q_{\text {ext }}=0.88$. In Fig. 14 (imaginary part) and Fig. 15 (real part) we show the variation of the frequency as we vary $y_{+}$.

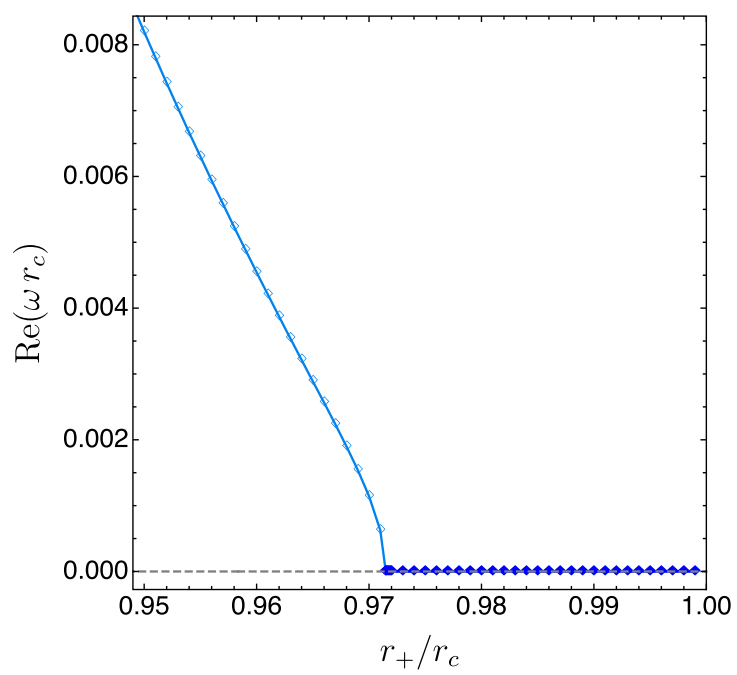

FIG. 15. Frequency spectrum (real part) for $q / q_{\mathrm{ext}}=0.88$ and $n=5, \ell=2$. The dark red "Black circle" and brown inverted triangle "Down-pointing black triangle" branches of Fig. 14 are not shown because they simply have $\operatorname{Re}\left(\omega r_{c}\right)=0$. 

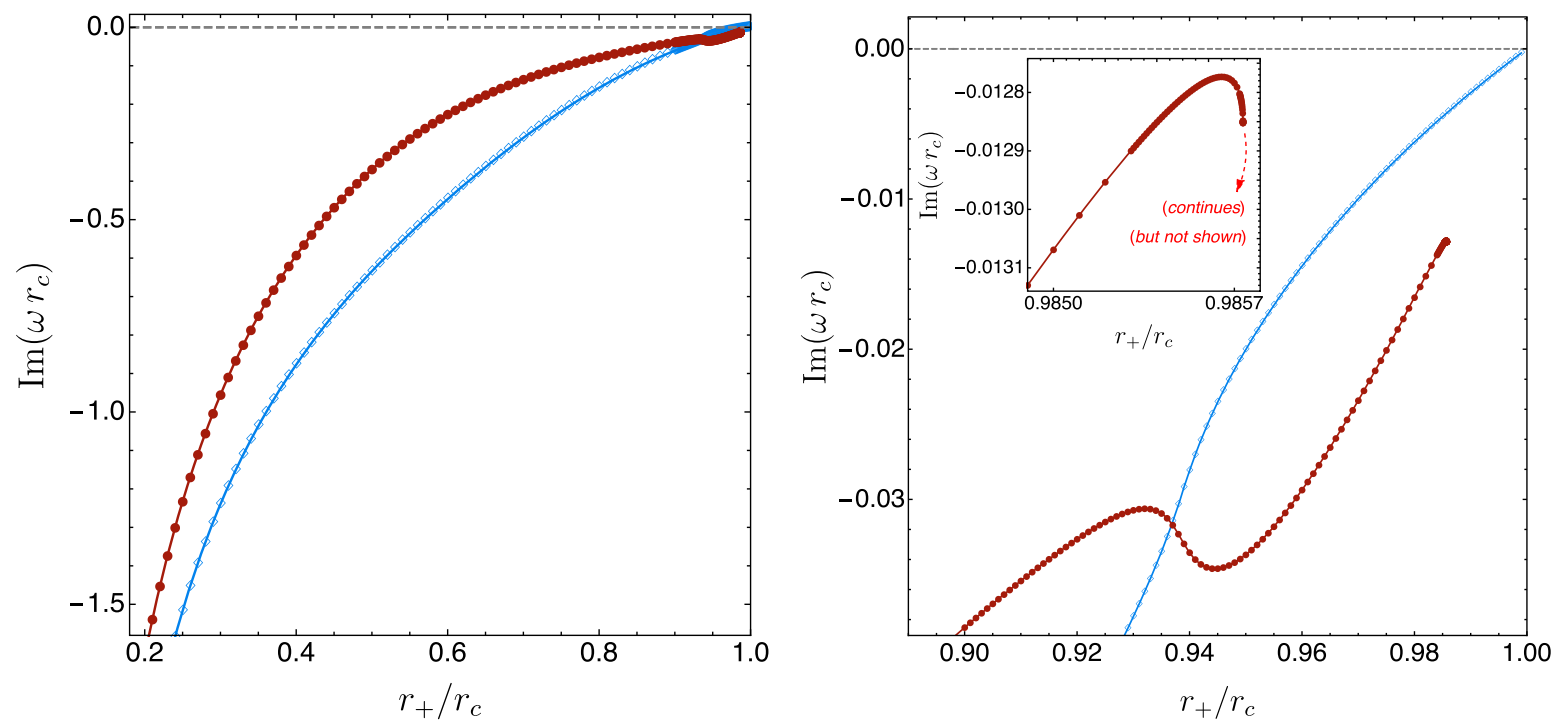

FIG. 16. Frequency spectrum (imaginary part) for $q / q_{\text {ext }}=0.85$ and $n=5, \ell=2$ (left panel) with zooms in relevant regions (right panel).

From Fig. 3 (green - curve) such black holes are stable for all values of $y_{+}$. It follows that the curve on the top of Fig. 14 with dark red disks $(\bullet)$ has $\operatorname{Im}\left(\omega r_{c}\right)<0$ for any $y_{+}$: this is the family of modes that becomes unstable but only for $q / q_{\text {ext }} \gtrsim 0.881$. Additionally, out of an infinite family of modes with $\operatorname{Im}\left(\omega r_{c}\right)<0$ for any $y_{+}$, we further show the families of modes that have the smallest $\left|\operatorname{Im}\left(\omega r_{c}\right)\right|$. This is the light blue $\diamond$ branch [with $\operatorname{Re}\left(\omega r_{c}\right) \neq 0$; see Fig. 15] that bifurcates at $q / q_{\text {ext }} \sim 0.971$ into two branches with $\operatorname{Re}\left(\omega r_{c}\right)=0$, namely, the blue diamond $(\checkmark)$ branch and the brown inverted triangle ( $\nabla)$ branch. A zoom in of the region close to $y_{+}=1$ is displayed in the right panel of Fig. 14. This plot complements previous plots at constant $y_{+}$(of Figs. 7, 9, and 11). For example, in the right panel of Fig. 14, we identify the upper three points $\bullet, \nabla, \nabla$ with $y_{+}=0.99$ which are the three points $\bullet, \boldsymbol{\nabla}, \boldsymbol{\nabla}$ with $q / q_{\text {ext }}=0.88$ in the left panel of Fig. 7 (which describes RNdS solutions with $y_{+}=0.99$; we do not show the green square family in Fig. 14). As another example, still in the right panel of Fig. 14, we identify the two points $\bullet, \diamond$ with $y_{+}=0.95$ that are the two points $\bullet, \diamond$ with $q / q_{\text {ext }}=0.88$ of Fig. 9 (which describes RNdS solutions with $y_{+}=0.95$; we do not show the orange triangle family in Fig. 14). As a final example, in the left panel of Fig. 14, we also identify the two points $\bullet, \diamond$, this time with $y_{+}=0.70$, which are the two points $\bullet, \diamond$ with $q / q_{\text {ext }}=0.95$ of Fig. 11 (which describes RNdS solutions with $\left.y_{+}=0.70\right)$.

(3) Finally, we considered RNdS black holes with $q / q_{\mathrm{ext}}=0.85$. In Figs. 16 and 17 we display the three family of modes with the lowest $\left|\operatorname{Im}\left(\omega r_{c}\right)\right|$ for this RNdS family. Such black holes are stable for all values of $y_{+}$(see green $\square$ curve in Fig. 3). In the main plot of the right panel, we identify the blue $\diamond$ point with $y_{+}=0.99$ which is also the blue $\diamond$ point with $q / q_{\text {ext }}=0.85$ in the left panel of Fig. 7 (which describes solutions with $y_{+}=0.99$ ). Also in the main plot of the right panel, we further identify the two points (blue $\diamond$ and dark red $\bullet$ ) with $y_{+}=0.95$ which are also the two points (blue $\diamond$ and dark red $\bullet$ ) with $q / q_{\text {ext }}=0.85$ in the right panel of Fig. 9 (which describes solutions with $y_{+}=0.95$ ). Finally,

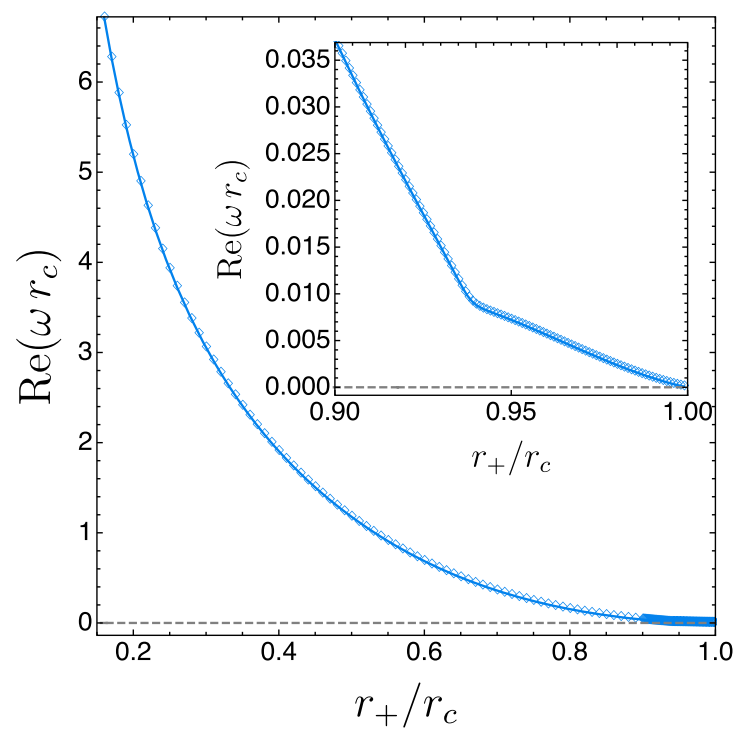

FIG. 17. Frequency spectrum (real part) for $q / q_{\text {ext }}=0.85$ and $n=5, \ell=2$. The dark red $\circ$ and green "Filled square" branches of Fig. 16 are not shown because they simply have $\operatorname{Re}\left(\omega r_{c}\right)=0$. 
in the left panel, we identify the two points (dark red - and blue $\diamond$ ) with $y_{+}=0.7$ which are the two points (dark red $\bullet$ and blue $\diamond$ ) with $q / q_{\text {ext }}=0.85$ of Fig. 11 (which describes solutions with $y_{+}=0.70$ ). Note that one of the two branches (blue $\diamond$ and dark red $\bullet$ ) has the lowest timescale depending on the window of $y_{+}$.

\section{DISCUSSION}

We believe that our study reveals key aspects of the gravitational instability of Reissner-Nordström de Sitter black holes originally found in [2] and further studied in $[4,6]$. The fundamental novel results that we establish can be summarized as follows:

(i) RNdS black holes are unstable when $d \geq 6$ (see, e.g., Fig. 2). For $d \geq 7$ this instability was first established in $[2,4,6]$. In addition, we find it is also present for $d=6$ (although with a much lower timescale).

(ii) We have established the physical origin of the instability: it is present because in the near-horizon limit of extremal RNdS, the unstable gravitational modes effectively behave as a massive scalar field whose mass violates the $\mathrm{AdS}_{2}$ BreitenlöhnerFreedman bound (if and only if $d \geq 6$; see Fig. 1). By continuity, the instability then extends away from extremality.

(iii) The instability criterion of the previous item is known as the Durkee-Reall conjecture [19] later proved by Hollands and Wald [20] when $\Lambda \leq 0$. Our findings provide good numerical evidence for the Durkee-Reall conjecture with de Sitter asymptotics $(\Lambda>0)$, as already argued in [20]. It would be interesting to formally prove this result for $\Lambda>0$ using the methods of [20].

(iv) Our results also confirm that the Durkee-Reall instability criterion $[19,20]$ provides a sufficient but not necessary condition for instability. For example, for extremal black holes the instability criterion typically predicts instability only for certain windows of the dimensionless horizon radii ratio $r_{+} / r_{c}$ (see Fig. 1) but we find that actually the instability is present for any value of this ratio (at extremality and for $d \geq 6$ ); see, e.g., Fig. 2. In particular, this also means that there is no critical minimum value of $y_{+}$for the existence of the instability unlike it was claimed in [3].

(v) RNdS black holes are parametrized by the dimensionless ratios $y_{+} \equiv r_{+} / r_{c}$ and $q / q_{\text {ext }}$. For a given dimension $n$ and $y_{+}$, we have found the onset charge $q^{\text {onset }} / q_{\text {ext }}$ above which RNdS becomes unstable; see Fig. 3.

(vi) In addition to finding the instability timescale at extremality (Fig. 2) and the onset charge (Fig. 3), we have spanned the two-dimensional parameter space of RNdS to produce the three-dimensional plot of Fig. 4 that displays the instability timescale as a function of $y_{+} \equiv r_{+} / r_{c}$ and $q / q_{\mathrm{ext}}$. In particular, as best seen in Fig. 5, after its onset the instability first increases as we approach extremality until it reaches a maximum near but before extremality. Then its strength decreases slightly as we approach further and reach extremality.

(vii) The instability is present for $d \geq 6$ for the harmonic mode $\ell=2$. Typically, the instability then tends to get weaker and even disappear as the harmonic number $\ell$ grows (see Fig. 6). For example, in $d=6$, 7 only the $\ell=2$ mode is unstable, in $d=8,9 \ell=3$ is also unstable, and in $d=10,11 \ell=2,3,4$ modes are unstable but not $\ell \geq 5$.

(viii) If we follow the unstable modes to regions of the parameter space where the instability shuts down and if we include the second and/or third families of quasinormal modes with lowest $\left|\operatorname{Im}\left(\omega r_{c}\right)\right|$ we find an intriguing network of mode bifurcations/mergers that seems to be absent in black holes with $\Lambda \leq 0$. This spectrum seems so unique and intriguing that we dedicated a special study to it. In the sequence of Figs. 7-8, 9-10, and 11 we have fixed representative values of $y_{+}$and analyzed how the frequency spectrum changes as the charge of the RNdS black hole varies. On the other hand, in Figs. 12-17 we fixed some values of $q / q_{\text {ext }}$ and changed $y_{+}$.

There are quite a few natural extensions of our work. In particular, it would be interesting to frame the quasinormal mode structure we found in this manuscript in light of the three families of quasinormal modes used to study the strong cosmic censorship conjecture [7] for initial data close to RNdS black holes [8-18]. We should note, however, that once the instability sets in, a novel black hole geometry is likely to form [22]. Strong cosmic censorship should then be studied by investigating how slowly generic perturbations decay around the new hypothetical background geometry. Away from extremality, we should be able to settle this issue by studying the quasinormal mode spectrum of the relevant RNdS black hole.

\section{ACKNOWLEDGMENTS}

We would like to thank V. Cardoso, R. A. Konoplya, and A. Zhidenko for reading an earlier version of this manuscript. O. J. C. D. is supported by the STFC Ernest Rutherford Grant No. ST/K005391/1, and by the STFC "Particle Physics Grants Panel (PPGP) 2016" Grant No. ST/P000711/1. J.E. S. is supported in part by STFC Grants No. PHY-1504541 and No. ST/P000681/1. J.E. S. also acknowledges support from a J. Robert Oppenheimer Visiting Professorship. This work used the DIRAC Shared Memory Processing system at the University of Cambridge, operated by the COSMOS Project at the Department of Applied Mathematics and Theoretical 
Physics on behalf of the STFC DiRAC HPC Facility (www .dirac.ac.uk). This equipment was funded by BIS National e-Infrastructure capital Grant No. ST/J005673/1, STFC capital Grant No. ST/H008586/1, and STFC DiRAC Operations Grant No. ST/K00333X/1. DiRAC is part of the National e-Infrastructure.
[1] H. Kodama and A. Ishibashi, Master equations for perturbations of generalized static black holes with charge in higher dimensions, Prog. Theor. Phys. 111, 29 (2004).

[2] R. A. Konoplya and A. Zhidenko, Instability of Higher Dimensional Charged Black Holes in the de-Sitter World, Phys. Rev. Lett. 103, 161101 (2009).

[3] R. Konoplya and A. Zhidenko, Instability of $D$-dimensional extremally charged Reissner-Nørdstrøm (-de Sitter) black holes: Extrapolation to arbitrary D, Phys. Rev. D 89, 024011 (2014).

[4] V. Cardoso, M. Lemos, and M. Marques, Instability of Reissner-Nordström black holes in de Sitter backgrounds, Phys. Rev. D 80, 127502 (2009).

[5] W.F. Buell and B. A. Shadwick, Potentials and bound states, Am. J. Phys. 63, 256 (1995).

[6] K. Tanabe, Instability of the de Sitter Reissner-Nordstrom black hole in the $1 / D$ expansion, Classical Quantum Gravity 33, 125016 (2016).

[7] R. Penrose, Singularities of spacetime, in Theoretical Principles in Astrophysics and Relativity, edited by W. R. N. R. Liebowitz and P. O. Vandervoort (Chicago University Press, Chicago, 1978), pp. 217-243.

[8] V. Cardoso, J. L. Costa, K. Destounis, P. Hintz, and A. Jansen, Quasinormal Modes and Strong Cosmic Censorship, Phys. Rev. Lett. 120, 031103 (2018).

[9] J. L. Costa, P. M. Girão, J. Natário, and J. D. Silva, On the occurrence of mass inflation for the Einstein-Maxwellscalar field system with a cosmological constant and an exponential Price law, Commun. Math. Phys. 361, 289 (2018).

[10] O. J. C. Dias, F. C. Eperon, H. S. Reall, and J. E. Santos, Strong cosmic censorship in de Sitter space, Phys. Rev. D 97, 104060 (2018).
[11] O. J. Dias, H.S. Reall, and J. E. Santos, Strong cosmic censorship: Taking the rough with the smooth, J. High Energy Phys. 10 (2018) 001.

[12] M. Dafermos and Y. Shlapentokh-Rothman, Rough initial data and the strength of the blue-shift instability on cosmological black holes with $\Lambda>0$, Classical Quantum Gravity 35, 195010 (2018).

[13] S. Hod, Strong cosmic censorship in charged black-hole spacetimes: As strong as ever, Nucl. Phys. B941, 636 (2019).

[14] O. J. Dias, H.S. Reall, and J. E. Santos, Strong cosmic censorship for charged de Sitter black holes with a charged scalar field, Classical Quantum Gravity 36, 045005 (2019).

[15] O. J. Dias, H. S. Reall, and J. E. Santos, The BTZ black hole violates strong cosmic censorship, J. High Energy Phys. 12 (2019) 097.

[16] H. Zhang and Z. Zhong, Strong cosmic censorship in de Sitter space: As strong as ever, arXiv:1910.01610.

[17] Y. Gim and B. Gwak, Charged particle and strong cosmic censorship in Reissner-Nordström-de Sitter black holes, Phys. Rev. D 100, 124001 (2019).

[18] H. Liu, Z. Tang, K. Destounis, B. Wang, E. Papantonopoulos, and H. Zhang, Strong cosmic censorship in higherdimensional Reissner-Nordström-de Sitter spacetime, J. High Energy Phys. 03 (2019) 187.

[19] M. Durkee and H. S. Reall, Perturbations of near-horizon geometries and instabilities of Myers-Perry black holes, Phys. Rev. D 83, 104044 (2011).

[20] S. Hollands and A. Ishibashi, Instabilities of extremal rotating black holes in higher dimensions, Commun. Math. Phys. 339, 949 (2015).

[21] O. J. C. Dias, J. E. Santos, and B. Way, Numerical methods for finding stationary gravitational solutions, Classical Quantum Gravity 33, 133001 (2016).

[22] O. J. C. Dias and J. E. Santos (to be published). 